Gewässerschutz bei Regenwetter ist ein Thema, dessen Bedeutung mit der Siedlungsdichte zunimmt. Darüber wurde bereits in der zweiten Ausgabe von GAIA vorwiegend aus der Sicht der Ingenieure berichtet ${ }^{[2]}$, und hier schließt sich nun eine Betrachtung der Gewässerbiologen an. Obschon die biologischökologischen Probleme im Zusammenhang mit Siedlungsentwässerung und Gewässerschutz heute erkannt sind, gibt es erst wenige Versuche, Zusammenhänge zwischen der Urbanisierung von Gewässern und deren "Biologie" aufzudecken. Die (Ingenieur-)Frage nach dem biologischen Effekt von Mischwassereinleitungen in Kleine Fließgewässer bei Regenwetter läßt sich daher nicht so einfach beantworten. Dennoch können in vielen Fällen bereits mit dem vorhandenen biologischen Fachwissen Gewässerschutzprobleme auch in Siedlungen ökologisch befriedigend gelöst werden - vorausgesetzt, es findet eine echte interdisziplinäre Zusammenarbeit statt, und Wissenschaft und Praxis finden zueinander.

\title{
Biologische Aspekte des Gewässerschutzes in urbanisierten Gebieten
}

\author{
Andreas Frutiger* und Sonja Gammeter
}

\begin{abstract}
In Europe more and more storage ponds are installed in sewer systems in order to prevent sewage water to get unpurified into the receiving water course during rainfall. Although this strategy is very expensive its ecological use has hardly been verified. Investigations in receiving rivers show that the biological effects of sewage pollution during storms are usually modest and often negligible compared to the impacts of morphological modifications of the river bed or the river bank and the pollutional effects of dry weather flow.

The benthic biocenosis of such running waters is often poor and consists of only few "generalist-species", which are typically very robust and hardly limited by short-time pollution during storms.

Yet no general concepts for an ecologically optimal strategy for urban drainage and waste water treatment system can be given. Since each species has its own ecological demands, which have to be fulfilled for it to survive in the river,

each river has to be treated individually. First its main problems (morphology? water quality? flow regime? et cetera) have to be identified and subsequently an optimal strategy should be defined.
\end{abstract}

Keywords: benthos, combined sewer overflows, pollution control,

rainfall, receiving waters, river management, urban drainage

* Postadresse : Dr. A. Frutiger Abteilung Hydrobiologie/Limnologie

Eidgenössische Anstalt für Wasserversorgung Abwasserreinigung und Gewässerschutz (EAWAG)

CH-8600 Dübendorf (Schweiz) stem zur biologischen Gütebeurteilung von abwasserbelasteten Fließgewässern entwickelt (das Saprobiensystem ${ }^{|3|}$ ), aber da sie es noch kaum vermochten, differenzierte Aussagen über die kausalen Zusammenhänge zwischen der Ursache (Belastung) und deren Wirkung (biologische Reaktion) zu machen, hatten ihre Arbeiten wenig Rückwirkung auf die Tätigkeit der Ingenieure.

Mit dem vorliegenden Artikel soll versucht werden, die Problematik der Siedlungsentwässerung aus der Sicht der Gewässerbiologen darzustellen. Dabei wird unter Hinweis auf biologische Grundsätze erläutert, warum die "Biologie" im Unterschied $\mathrm{zu}$ ingenieurtechnischen Systemen - einheitliche Vorgehensweisen oder Lösungen nicht zuläßt.

Aus ökonomischen und betriebstechnischen Gründen werden Kläranlagen so dimensioniert, daß sie das Abwasser nicht "immer", sondern nur "meistens", das heißt während etwa 95 Prozent der Zeit, aufnehmen können. Wollte man die Anlagen erweitern, so daß sie alles Abwasser praktisch immer aufnehmen könnten, würde dies die Kosten etwa um den Faktor 10 erhöhen! Es wird also bewußt in Kauf genommen, daß während etwa 400 Stunden im Jahr die Kläranlagen für die momentane Abwassermenge nicht mehr ausreichen und über sogenannte Regenüberläufe ungereinigtes, aber durch Regenwasser stark verdünntes Abwasser ("Mischwasser") in den Vorfluter gelangt Dieses Konzept spiegelt sich unter anderem auch in der "Verordnung über Abwassereinleitungen" [4] wider, deren Anforderungen für Einleitungen in ein Gewässer nur bei Trockenwetter gelten.

Es waren vor allem Zuflußuntersuchungen an Seen, welche Zweifel am Sinn dieses Entwässerungskonzepts aufkommen ließen. So wurde gefunden, daß in den kritischen 5 Prozent der Zeit (das heißt während Regenereignissen) ein erheblicher, oft sogar der Löwenanteil der Nähr- beziehungsweise Schadstofffracht in den Vorfluter gelangt, besonders mit dem ersten Mischwasserstoß. Da Untersuchungen an Fließgewässern zu ähnlichen Befunden führten und zudem zeigten, daß nicht nur die Fracht, sondern auch die Schadstoffkonzentrationen der ersten Mischwasserwelle bedenklich hoch sein können (vergleiche Figur 1), begann man, die Kanalisationsnetze mit Mischwasserspeichern zu erweitern. Diese sollen mindestens den ersten, besonders schadstoffreichen Mischwassersto $\boldsymbol{B}$ auffangen, um ihn nach dem Regenereignis dosiert über die Kläranlage abfiihren zu können.

Derartige Schmutzwasserwellen können für das Fließgewässer in verschiedener 


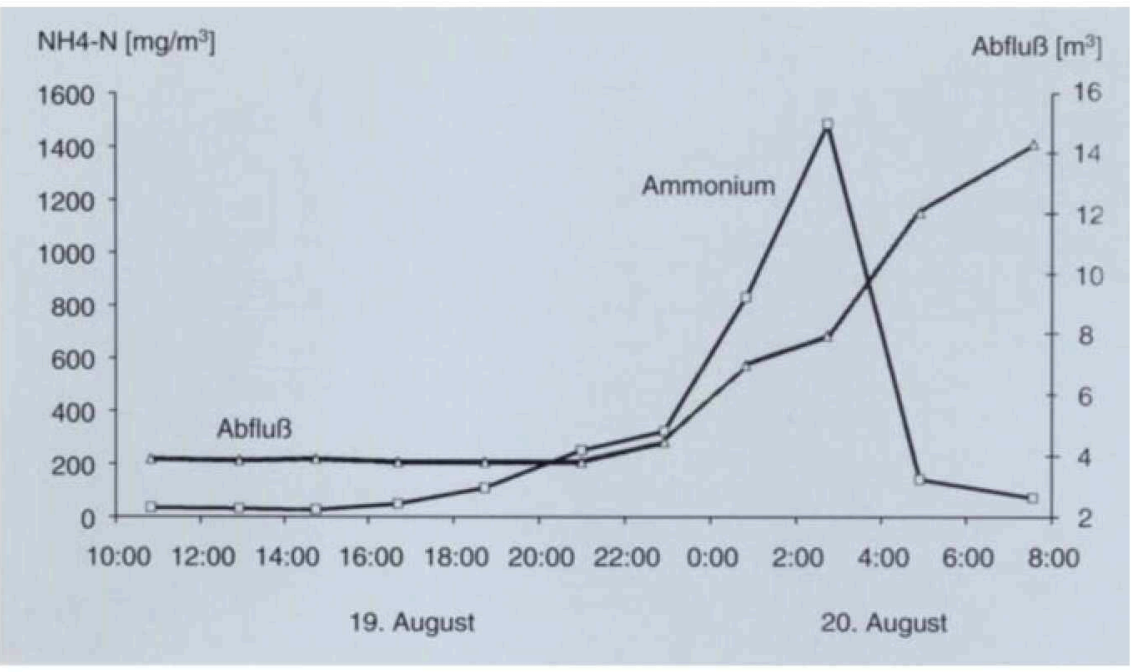

Figur 1. Ein kurzes Regenereignis über dem Toggenburg am 19. August 1985 hat zur Folge, daß durch einen Mischwasser-Überlauf bei der Kläranlage Wattwil ungereinigtes Abwasser in die Thur gelangt. Die Ammonium-Spitze erscheint einige Stunden vor dem Abflußmaximum in der Thur, da das Regenwasser aus dem Siedlungsgebiet viel schneller abfließt als aus dem natürlichen Einzugsgebiet [5]. - Vergleiche dazu auch entsprechende Ergebnisse aus der Fallstudie "Fehraltorf" im Bericht (dort Figur 9) von Krejci, Lange und Schilling [2].

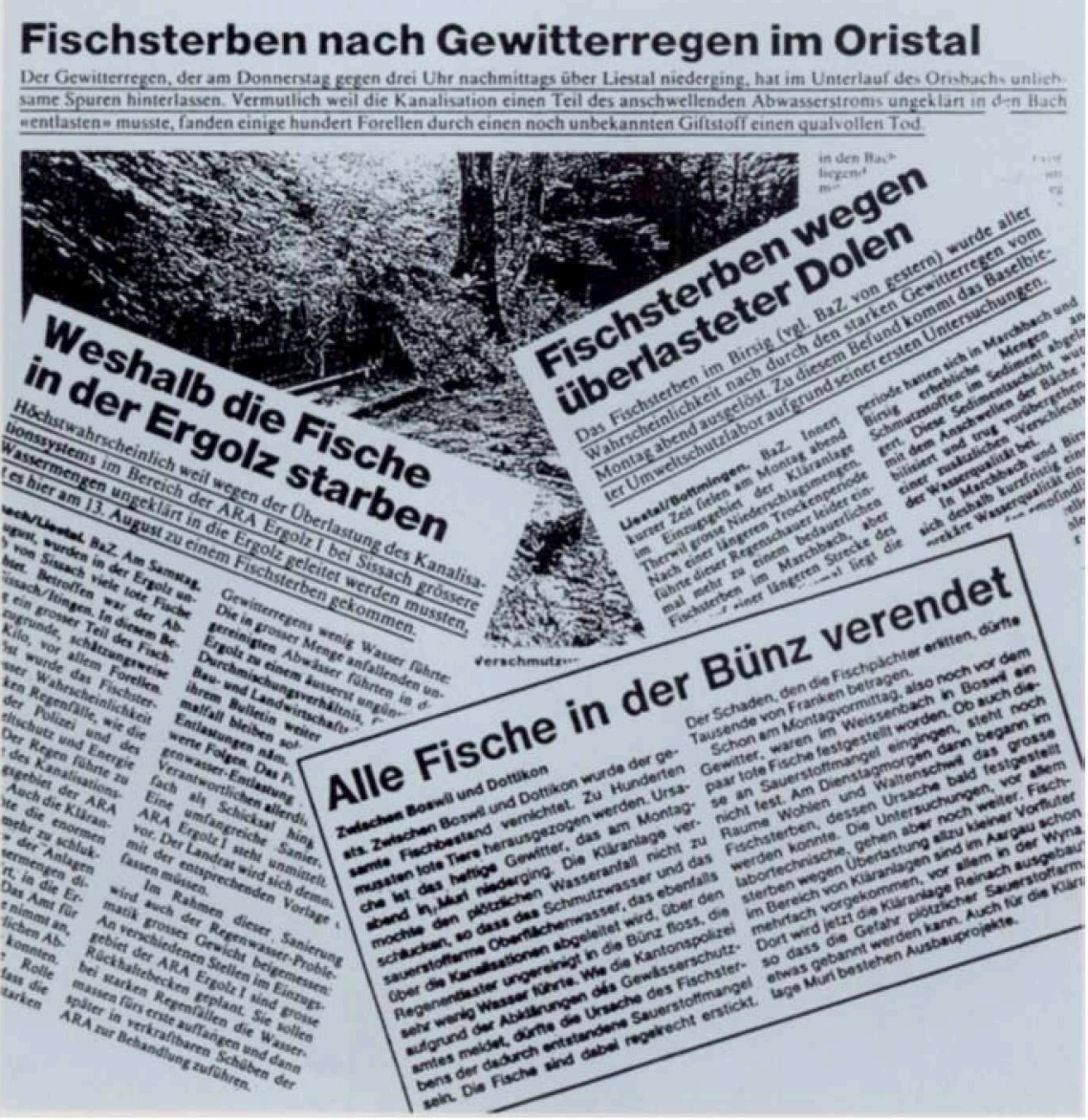

Figur 2. Fischsterben (vor allem Forellen) in der Folge von Gewitterregen treten im Sommer immer wieder auf, besonders in kleinen Fließgewässern nach langen Trockenzeiten, da die Fische durch die geringe Wasserführung (Strömung) und hohe Temperaturen schon stark in Mitleidenschaft gezogen sind. Das Mischwasser bringt schließlich "das Faß zum Überlaufen", mithin beginnt das "publikumswirksame" Fischsterben.
Weise schädlich sein; am besten bekannt sind wohl die immer wieder vorkommenden Fischsterben, die sehr oft durch einen gewitterartigen Regen ausgelöst werden (Figur 2). Bevor aber darauf näher eingegangen werden kann, sollen die wesentlichen Charakteristika des Lebensraumes "Fließgewässer" skizziert werden.

\subsection{Lebensraum "Fließgewässer"}

Fließgewässer sind nicht bloß Rinnen, in denen Wasser abfließt. Sie sind auch Lebensräume, welche von einer Vielzahl verschiedenster Organismen besiedelt werden. Neben der Fischbesiedlung (in einem unbeeinträchtigten Bach bis zu einem Dutzend verschiedener Arten) finden sich auf jedem Quadratmeter Bachsohle zwischen 5000 und 100000 Kleintiere (Insektenlarven, Kleinkrebse, Milben, Würmer und so weiter) aus mehreren Hundert Arten. Viele dieser Tiere zeichnen sich durch eine hohe bis extreme Anpassung an die im Bach herrschenden Verhältnisse aus, vor allem an den permanenten hydraulischen Streß, an die Sedimentbewegungen bei Hochwasser ("Geschiebetrieb") und an die zuverlässigen Austausch- und Mischungsprozesse. Diese weitgehende Spezialisierung der Organismen ist im Zusammenhang mit Gewässerschutzfragen deshalb besonders relevant, weil sie zur Folge hat, daß die Organismen eine entsprechend geringe Toleranz gegenüber anthropogenen Veränderungen aufweisen und schon bei geringfügigen Abweichungen von dem Zustand, an den sie angepaßt sind, in Schwierigkeiten geraten. Besonders empfindlich sind sie gegen Änderungen von Strömung, Sohlenbeschaffenheit, Sauerstoffgehalt und Temperatur.

Der Lebensraum "Fließgewässer" umfaßt neben dem fließenden Wasser auch die Bachsohle mit dem darunterliegenden Sedimentkörper samt seinem Lückenraum ("Interstitial") und die Uferstreifen mit ihrer Vegetation. Die ökologische Bedeutung dieser einzelnen Teilhabitate ist in Figur 3 am Beispiel der Eintagsfliege Ecdyonurus dargestellt. Die Eier und auch die kleinen Larven von Ecdyonurus entwickeln sich im Interstitial in einer Tiefe von bis zu einem halben Meter. Dort sind sie gegen die Strömung und gegen die Gefahren des Geschiebetriebs bei Hochwasser geschützt. Die kleinen Larven ernähren sich von feinem Detritus (hauptsächlich Pflanzenreste), der in die Lückenräume eingeschwemmt wird, und vom Bakterienfilm, welcher jeden Stein und jedes Sandkorn überzieht. Wenn sie 


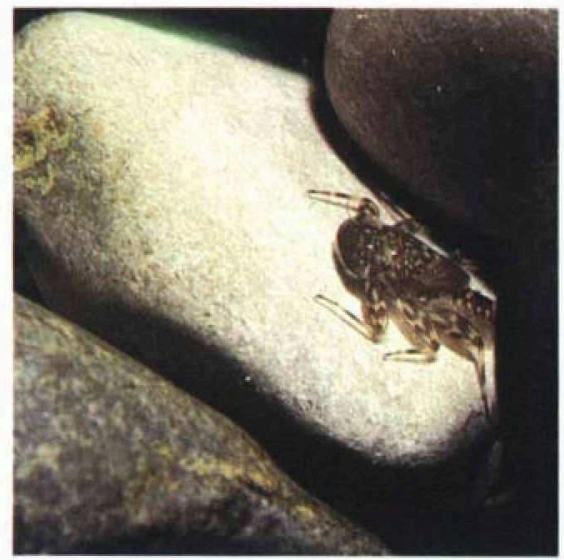

Ecdyonurus-Larve: Die Eintagsfliege Ecdyonurus, ein sehr häufiger Bewohner von Fließgewässern, verbringt einen großen Teil ihres Lebenszyklus als Larve auf und in der Sohle von Bächen,

zuerst in den tieferen Schichten der Sohle, in späteren Wachstumsstadien auf der Oberseite der Steine (vergleiche auch Figur 3).

größer werden, begeben sie sich an die Oberfläche der Bachsohle und weiden den Algenbelag von der Oberseite der Steine ab. Ihre stark abgeplattete Körperform ist eine direkte biologische Anpassung an diese Emährungsweise. Am Ende ihrer larvalen Entwicklung begeben sich die Tiere in strömungsarme Zonen in Ufernähe und verlassen das Wasser (Emergenz). Das nun flugfähig gewordene Tier sucht sofort Schutz in der Ufervegetation, um sich ein letztes Mal zu häuten. Nach wenigen Stunden bis Tagen ist Ecdyonurus geschlechtsreif und fliegt auf, um sich im Flug zu paaren und kurz darauf zu sterben (daher der Name "Eintagsfliege"). Die begatteten Weibchen streifen ihre Eier an der Wasseroberfläche des Baches ab, von wo diese ins Interstitial der Gewässersohle absinken. Damit beginnt der Entwicklungszyklus von neuem. Die Entwicklung vieler anderer Fließwassertiere verläuft ähnlich wie diejenige von Ecdyonurus und ist nur dann erfolgreich, wenn sämtliche Teilhabitate vorhanden und bewohnbar sind.

Unter biologischem Aspekt bedeutsam ist die Tatsache, daß viele Fließgewässer immer wieder durch Hochwasser mit Geschiebetrieb gestört werden. Dabei wird oft ein großer Teil des "Benthos" (Tierund Pflanzenbesiedlung der Gewässersohle) vernichtet. Nach jedem derartigen Ereignis setzt eine Wiederbesiedlung mit einer charakteristischen Abfolge von Pionierformen ( $r$-Strategen) über Nachfolgeformen bis zu Klimaxformen (k-Strategen) ein. Daher findet man in natürlichen Fließgewässern neben vielen Arten mit einem einjährigen Entwicklungszyklus (Synchronisation mit der Jahreszeit) und denjenigen mit einer vergleichsweise langen Entwicklungszeit (mehrere Jahre) auch solche mit einer extrem kurzen Generationsfolge (Tage bis Wochen). Letztere werden durch häufige Störungen (zum Beispiel Hochwasser) selektiv gefördert.

Figur 3. Der Lebensraum "Fließgewässer" umfaßt neben dem fließenden Wasser und der Gewässersohle auch den Porenraum der tieferen Sedimentschichten

(das sogenannte hyporheische Interstitial) und den angrenzenden Uferstreifen mit seiner Vegetation.

Wie die Eintagsfliege Ecdyonurus durchlaufen auch viele andere Fließwassertiere die unterschiedlichen

Phasen ihres Entwicklungszyklus in verschiedenen Teilhabitaten.

Nur wenn alle Teilhabitate vorhanden sind können diese Tiere ihren

Entwicklungszyklus beenden und sich im Gewässer natürlicherweise fortpflanzen

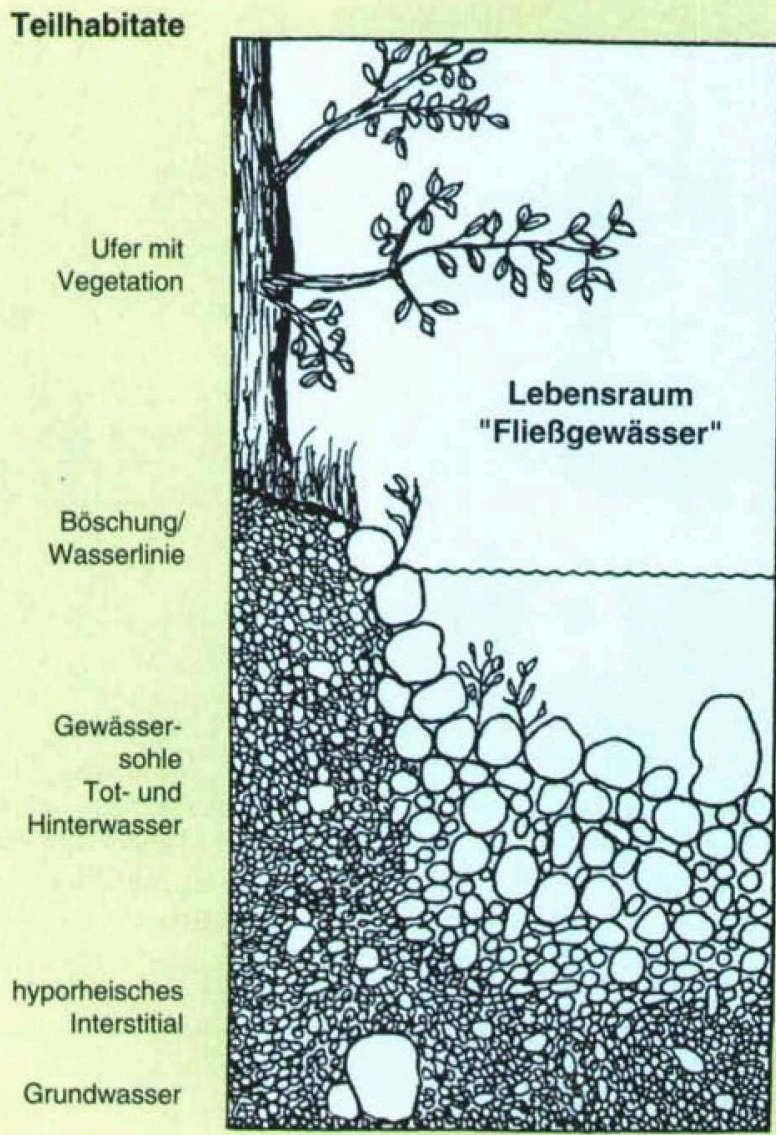

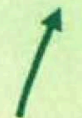

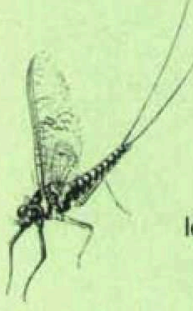

\section{Entwicklung von \\ Ecdyonurus}

Emergenz (aquatisch $\rightarrow$ terrestrisch)

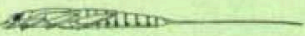

Reifung/ letzte Hâutung

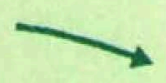

Partnersuche Kopulation
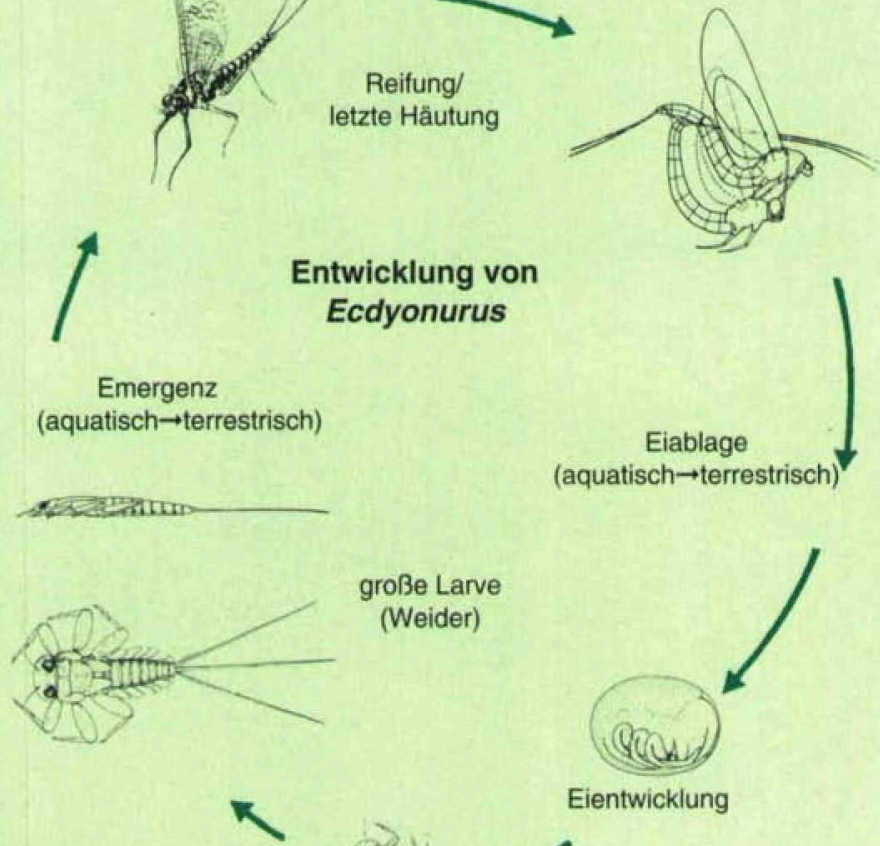

große Larve

(Weider)

kleine Larve

(detritivor) 


\subsection{Die Biozönose eines Gewässers als Indikator für dessen Zustand (Schädigung)}

Soll die Gewässerschutzstrategie über das Ende der Kanalisationsröhre hinausreichen und die Situation des Vorfluters in das Entwässerungskonzept einbezogen werden, kommt man nicht umhin, konkrete oder mögliche Schädigungen, welche durch Abwasserbelastungen im Gewässer entstehen, erfassen zu können. Dazu eignet sich die benthische Biozönose (Lebensgemeinschaft der Gewässersohle) besonders gut. Jeder Organismus, der sich auf oder in der Gewässersohle entwickelt, ist ja während seiner gesamten Aufenthaltszeit im Bach den dort herrschenden Bedingungen ausgesetzt und "integriert" daher gewissermaßen das chemische und physikalische Geschehen im Bach im Verlaufe seiner Entwicklung. Das Vorkommen der Art im Gewässer zeigt, daß - mindestens an gewissen Stellen - alle ihre Ansprüche an die Umwelt in bezug auf sämtliche relevanten Parameter (physikalische und chemische Bedingungen, Nahrungsgrundlage und so weiter) während ihrer gesamten Entwicklung erfüllt sind. In beeinträchtigten Gewässern verschwinden zuerst und vor allem diejenigen Arten, welche eine lange Entwicklungszeit brauchen. An ihre Stelle treten die typischen Pionierformen, weil diese rasch auf die neue (gestörte) Situation reagieren können. Die benthische Biozönose stellt somit, quasi als Endresultat eines stets natürlicherweise ablaufenden Bioassays, das gewaltige Informationspotential aller individuellen Einzelintegrale dar. Also kann die Veränderung einer Biozönose infolge eines anthropogenen Eingriffs als direktes $\mathrm{Maß}$ für die dadurch bewirkten Effekte verwendet werden.

\subsection{Welche Effekte sind durch kurze, stoßartige Schadstoffbelastungen zu erwarten?}

Einige mögliche Effekte im Gewässer durch stoßartige Schadstoffbelastungen lassen sich relativ leicht prognostizieren. So gelangt zum Beispiel mit dem Mischwasser in der Regel eine große Menge Ammonium $\left(\mathrm{NH}_{4}^{+}\right)$ins Gewässer, welches aus häuslichen Abwässern stammt (vergleiche Figur 1). Davon sind normalerweise rund 6 Prozent "deprotoniert", das heißt liegen als giftiges Ammoniak $\left(\mathrm{NH}_{3}\right)$ vor, wobei dieser Anteil aber bei ungewöhnlich hohem $\mathrm{pH}$-Wert bis gegen 20 Prozent des Ammoniums steigen kann. Da aus Laboruntersuchungen [10], bekannt ist, wie lange die Forellen welche Ammoniakkonzentrationen ertragen können, lassen sich für MischwasserÜberläufe in Abhängigkeit von der Wasserführung (Verdünnung) und von der Dauer des Ereignisses konkrete Ammonium-Grenzwerte ableiten, die nicht überschritten werden dürfen. Die im $\mathrm{Zu}$ sammenhang mit Mischwasserüberläufen auftretenden Fischsterben sind jedoch nicht immer durch eine toxische Ammoniakkonzentration zu erklären. Es sind noch etliche andere Faktoren mit im Spiel, welche sich zum Teil gegenseitig beeinflussen oder sogar verstärken (SynergieEffekte). Beispielsweise kann der Mischwassersto $\beta \mathrm{zu}$ einer signifikanten Verringerung des Sauerstoffgehalts im Vorfluter fuihren, sei es direkt durch Eintrag sauerstoffzehrender organischer Verbindungen und die relativ hohe Temperatur des Mischwassers oder indirekt dadurch, daß organische Partikeln, welche vor dem Regenereignis auf der Flußsohle abgelagert wurden, nun aufgewirbelt werden und dadurch eine Sauerstoffzehrung einsetzt. Somit wird die Versorgung der Tiere mit Sauerstoff ausgerechnet in einer Situation kritisch, wo sie ihn besonders nötig hätten (zur biochemischen Entgiftung von Ammoniak wird sehr viel Sauerstoff benötigt).

Für mehrere Organismenarten der Gewässersohle konnte die erhöhte Anfälligkeit gegen Ammoniak bei gleichzeitig reduziertem Sauerstoffgehalt im Laboratorium nachgewiesen werden $[11,12]$. Es wird aber kaum je möglich sein, solche Experimente für alle Arten der benthischen Biozönose durchzuführen. Dies wäre jedoch eine Voraussetzung für zuverlässige Prognosen über die Wirkung einer kurzfristigen Ammoniakbelastung für die gesamte Bachbesiedlung.

Die Aussagekraft von Laborexperimenten wird weiter relativiert durch die Tatsache, daß bei einem Regenereignis neben den beiden bisher erwähnten "Streßfaktoren" Ammoniak und Sauerstoffmangel in der Regel noch viele andere Einflußgrößen ebenfalls beruicksichtigt werden müssen:

- Je nach Intensität und Dauer des Regenereignisses laufen große Mengen stark verdïnnten Mischwassers in den Vorfluter $\left(Q_{\text {Überlauf }} \gg Q T W_{\text {Bach }}\right)$ oder aber geringere Mengen, wobei das Abwasser durch abfließendes Regenwasser nur um einen Faktor 3-4 verdünnt sein kann.

- Im ersten Fall können die Tiere durch die plötzlich erhöhte Strömungsgeschwindigkeit vom Untergrund weggerissen und so aus dem Bach ausgeschwemmt werden (sogenannte "hydraulische Belastung") um so mehr, wenn ihr Verhalten und ihre Reaktionsfähigkeit durch Gifteinwirkung bereits geschädigt sind ${ }^{[12]}$.
- Im zweiten Fall (oder generell in langsam fließenden Gewässerabschnitten) lagern sich organische Partikeln oder solche, die Schwermetalle oder andere Schadstoffe enthalten, auf und in der Bachsohle ab und bewirken dort eine chronische Vergiftung oder eine verminderte Sauerstoffversorgung (als Folge von Abbauprozessen oder verringerter Durchspülung des Interstitials).

- In Gewässern, deren Algen- oder Makrophytenwachstum durch das Nährstoffangebot limitiert ist, kann durch das vermehrte Angebot während Mischwasserüberläufen die Pflanzenproduktion stark erhöht werden (Eutrophierung), wodurch wiederum eine Reihe von Parametern (Strömung, Licht, pH-Wert, Sauerstoffgehalt und andere) beeinflußt wird.

- Die Wirkung von Giften ist zum Teil stark von den momentanen Bedingungen abhängig; zum Beispiel nimmt die Toxizität von Ammoniak mit steigender Temperatur zu. Daher ist zu erwarten, daß die gleiche Ammoniakbelastung im Sommer einen deutlich größeren Effekt hat als im Winter.

- Dadurch, daß sich die Tiere im Bachbett entwickeln und so jede Art während einer gewissen Zeit als Ei, als Eilarve, als Larve, als Puppe und zum Teil auch als ausgewachsenes Tier vorkommt, ändert sich die Besiedlung des Bachs permanent. Weil nicht jedes Entwicklungsstadium gleich empfindlich gegen verschiedenartige Belastungen ist, hängt der Effekt eines Regenereignisses stark davon ab, wann dieses eintritt.

\section{Das Projekt "Fehraltorf"}

Alle diese Überlegungen zeigen, daß sich viele ökologische Fragen im Zusammenhang mit einer modernen, auf den gesamtheitlichen Gewässerschutz ausgerichteten Siedlungsentwässerungs- und Abwasserentsorgungsstrategie nur durch eine ausgewogene Kombination von biologischen und ingenieurtechnischen Untersuchungen im Feld beantworten lassen.

Deshalb konzipierte die interdisziplinär zusammengesetzte Arbeitsgruppe "Siedlungshydrologie" an der Eidgenössischen Anstalt für Wasserversorgung, Abwasserreinigung und Gewässerschutz (EAWAG) im Jahre 1989 das Projekt "Fehraltorf", bei dem sie von der Gemeinde Fehraltorf, dem Amt für Gewässerschutz und Wasserbau des Kantons Zürich (AGW) sowie vom Bundesamt für Umwelt, Wald und Landschaft (BUWAL) unterstützt wird. Das generelle Ziel dieses Projekts ist es, an einem konkreten Fallbeispiel alle re- 
levanten Aspekte der modernen Siedlungsentwässerung $\mathrm{zu}$ berücksichtigen, welche sich auf dem Weg des Abwassers von dessen Entstehung im Einzugsgebiet bis zu dem Punkt auftun, wo es das Einzugsgebiet im Fließgewässer wieder verläßt, um somit zu einer integrierten Problemlösung zu gelangen.

Vor allem aus organisatorischen und ingenieurtechnischen Gründen bot sich die knapp 4000 Einwohner zählende Gemeinde Fehraltorf im Kanton Zürich als Untersuchungsgebiet an. Sie befindet sich in der Nähe der EAWAG (15 km) und besitzt ein einfach gegliedertes Entwässerungssystem mit 5 Mischwasserüberläufen unterschiedlichster Bauart. Zudem sind verschiedene $\mathrm{MeB}$ - und Steuerungseinrichtungen im Kanalnetz bereits vorhanden.

\subsection{Teilprojekt "Gewässerschutz bei Regenwetter"}

Von den Teilprojekten, in die das Projekt "Fehraltorf" gegliedert ist, behandelt der vorliegende Bericht nur das biologisch-ökologische Teilprojekt "Gewässerschutz bei Regenwetter". Dessen Zielsetzung ist es, anhand einer detaillierten Felduntersuchung die gesamtheitlichen Auswirkungen der im Untersuchungsgebiet stattfindenden Mischwassereinleitungen auf den Vorfluter zu erfassen und daran die Relevanz der in Abschnitt 1.3 beschriebenen Ursache-Wirkungsbeziehungen für ein bestimmtes Gewässer zu prüfen. Die daraus gewonnenen Erkennt- nisse sollen sowohl in den Entwässerungskonzepten von Gemeinden als auch beim Vollzug des Gewässerschutzgesetzes berücksichtigt werden.

\subsection{Der untersuchte Bach "Luppmen"}

Als Vorflutgewässer für die Siedlungsentwässerung dient die Luppmen, ein kleiner Bach (vergleiche Figur 4): Das gesamte Einzugsgebiet der Luppmen (bis unterhalb Fehraltorf) ist ungefähr 24 Quadratkilometer groß und erstreckt sich auf einer Höhe von 920 bis 520 Meter über dem Meeresspiegel. Oberhalb der Ortschaft Pfäffikon ist die Luppmen zu einem Weiher aufgestaut, dem Tobelweiher. Unterhalb dieses Weihers beginnt der als Untersuchungsgebiet ausersehene Bachabschnitt, dessen durchschnittliches Gefälle 1.5 Prozent beträgt.

Unterhalb des Tobelweihers kann die Luppmen aus ökologisch-morphologischer Sicht in drei Abschnitte unterteilt werden, nämlich in eine Referenzstrecke, eine Restwasserstrecke und eine urbanisierte Strecke:

Abschnitt I, Referenzstrecke (Tobelweiher bis Ableitung Dorfbach, etwa $0.5 \mathrm{~km}$ ): Vom Weiher aus fließt die Luppmen zunächst im natürlichen Bett durch bewaldetes Gebiet.

Abschnitt II, Restwasserstrecke (Ableitung Dorfbach bis Ortseingang Fehraltorf, etwa $3 \mathrm{~km}$ ): Über einen Schieber wird ein Teil des Wassers (je nach Wasserführung
10-350 Liter pro Sekunde, früher für Mühlebetrieb genutzt) in ein anderes Einzugsgebiet geleitet. In der Luppmen verbleibt bei Trockenwetter oft kein Wasser mehr, oder nur noch so wenig, daß es schon weit oberhalb Fehraltorf vollständig versickert. Zwischen Pfäffikon und Fehraltorf wird intensive Landwirtschaft betrieben; der Bachlauf ist mehr oder weniger stark begradigt und verbaut (Trapezprofil, Sohlschwellen, Sohlenpflästerung, Abstürze, Ufersicherung) und nur teilweise durch Ufergehölz beschattet. Da dieser Abschnitt praktisch nicht besiedelt ist, wurde er von den regelmäßigen Untersuchungen ausgespart.

Abschnitt III, urbanisierte Strecke (im Siedlungsgebiet von Fehraltorf, etwa $1.5 \mathrm{~km}$ ): Beim Ortseingang Fehraltorf mündet ein verdolter, ständig wasserführender Drainagegraben in die Luppmen, die hier in gleicher Weise verbaut ist wie im Abschnitt II. Daher führt die Luppmen innerhalb des Siedlungsgebiets Fehraltorf so gut wie ständig Wasser, abgesehen von extrem langen Trockenwetterperioden (wie im Sommer/Herbst 1991). Bis zum ersten Mischwasserüberlauf (im folgenden abgekürzt mit MÜ 1 bezeichnet) ist die Luppmen nicht durch Abwassereinflüsse belastet und das Wasser deshalb relativ "sauber". Auf der Höhe des zweiten Überlaufs MÜ 2 beginnt dann eine Teilstrecke mit Grundwasserexfiltration, da die (künstlich eingetiefte) Luppmen hier

Figur 4. Die Luppmen und inr Einzugsgebiet bei Pfäffikon (Abschnitt I) und Fehraltorf (Abschnitte II und III) mit den Probenahmestellen.

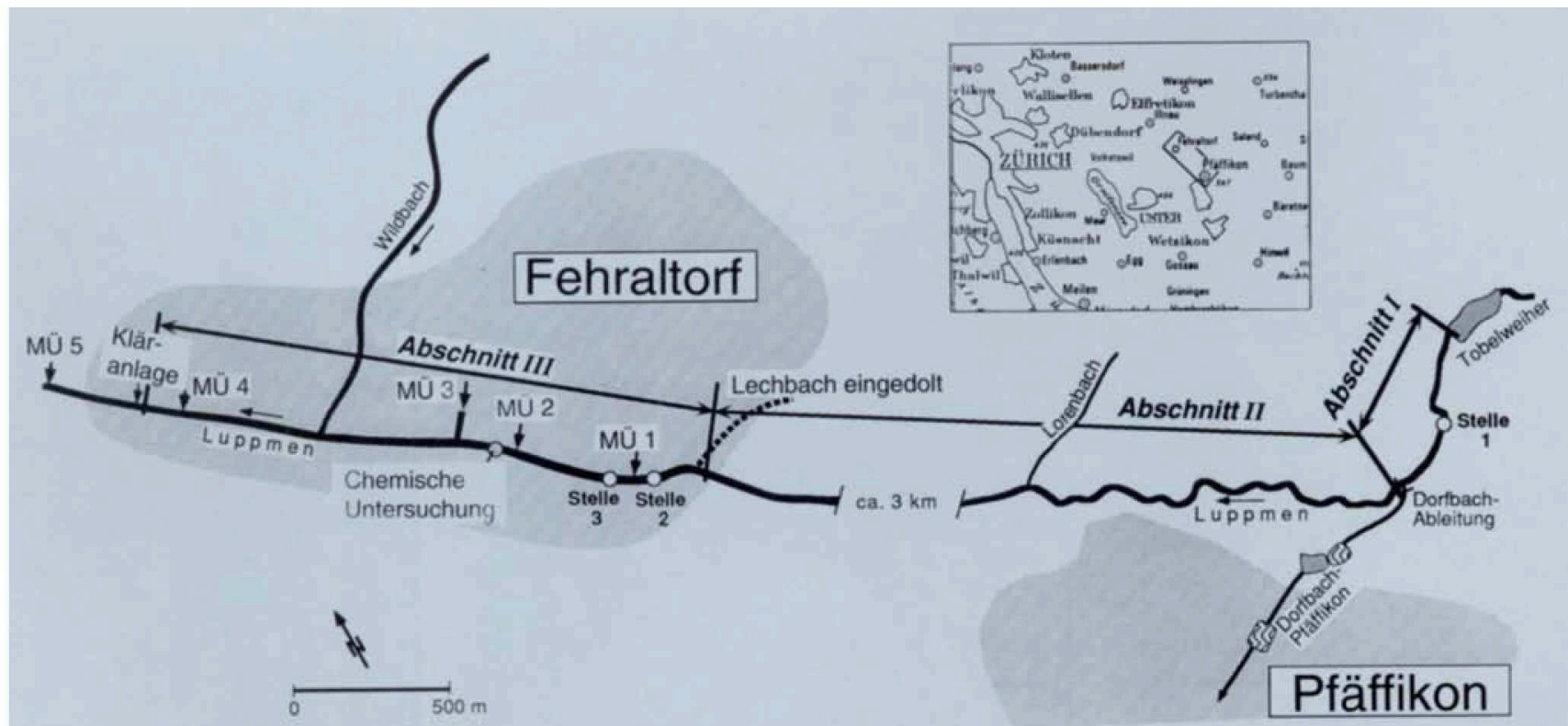


unterhalb des Grundwasserspiegels liegt (wiederum mit Ausnahme extremer Trokkenwetterperioden). Vor der Kläranlage am Ende des urbanisierten Abschnitts münden noch zwei weitere Mischwasseruiberläufe (MÜ 3, MÜ 4), einige Drainageleitungen und der "Wildbach" in die Luppmen.

\subsection{Meßgrößen und Methoden}

Zur Charakterisierung der in Abschnitt 1.3 erwähnten potentiellen Wirkungsparameter wird automatisch registriert, wann und wie lange die Mischwasserüberläufe anspringen und wie groß die Entlastungsmenge ist. Unterhalb von MÜ 2 werden zudem kontinuierlich die Konzentrationen von Ammonium und Sauerstoff sowie der $\mathrm{pH}$-Wert und die Temperatur gemessen. Die für die biologische Situation wichtigen Einflußgrößen Strömung, Strömungsverteilung, Temperatur, Korngrößenverteilung und Sohlenbeschaffenheit werden an mehreren Stellen erfaßt, der Abfluß und die Temperatur kontinuierlich. Im Sommer 1991 wurde in größeren Zeitabständen und einmal nach einem Überlauf-Ereignis aus drei Tiefen Interstitialwasser entnommen und analysiert (Ammonium, Sauerstoff, pH, Temperatur).

Um die tierische Besiedlung der Flußsohle zu erfassen, wurden kiesgefüllte, für die Kleintiere durchlässige Behälter (durchlöcherte Polyethylen-Flaschen, Volumen 1 Liter) in der Bachsohle inkubiert. Nach mindestens vier Wochen wurden die Behälter entnommen und die darin angesiedelten Tiere im Laboratorium gezählt und bestimmt. Auf diese Weise kann die Artenzusammensetzung und die Besiedlungsdichte der Benthosfauna über eine längere Zeitspanne alle ein bis zwei Wochen untersucht werden, ohne sie gravierend zu stören.

\section{Beobachtete Effekte der chemischen Belastung}

\subsection{Ammonium/Ammoniak}

Bei Trockenwetter liegt die Konzentration von Ammonium im Bach immer unterhalb des meßbaren Bereichs. Bei Überlauf-Ereignissen hingegen, welche in der Regel zwischen einer und drei Stunden dauem, wurden $\mathrm{NH}_{4}^{+}$-Konzentrationen zwischen 0.1 und $1.5 \mathrm{Gramm}$ pro Kubikmeter gemessen $\left(\mathrm{NH}_{3}\right.$ maximal $0.03 \mathrm{~g} / \mathrm{m}^{3}$ ). Diese Werte liegen weit unter den für Bachforellen kritischen Werten und sind somit für die Biozönose vermutlich kaum relevant, da alle uibrigen Tiere, von denen es diesbezügliche toxikologische Kenntnisse gibt, wesentlich weniger empfindlich sind als die Bachforelle. Ein extremes Ereignis allerdings bewirkte, daß während zehn Stunden eine $\mathrm{NH}_{4}^{+}$-Konzentration von $5.5 \mathrm{~g} / \mathrm{m}^{3}\left(\mathrm{NH}_{3}\right.$ $0.3 \mathrm{~g} / \mathrm{m}^{3}$ ) bestehen blieb: Ein kurzer, heftiger Regen nach einer mehrwöchigen Trockenperiode führte gerade noch $\mathrm{zu}$ einem Überlauf des Mischwasserspeichers. Das nur wenig verdünnte Abwasser floß ins völlig ausgetrocknete Bachbett, wo es sich in Pfützen sammelte und allmählich versickerte. Das Abwasser wurde erst durch ein weiteres Regenereignis zehn Stunden später wieder aus dem Bach ausgeschwemmt. Diese Situation $\left(0.3 \mathrm{~g} \mathrm{NH}_{3}\right.$-Stickstoff $/ \mathrm{m}^{3}$ während 10 Stunden) ist für Forellen durchaus kritisch: Das einzige im gesamten Bachabschnitt verbliebene Wasser, in das sich sämtliche Fische vor der allmählichen Austrocknung geflüchtet hatten, war das unter einer Brücke gelegene, künstliche Becken, in welches der Mischwasserüberlauf einmündet; - nur wenige überlebten (Figur 5a).

\subsection{Sauerstoff}

Im freien Wasser (der sogenannten "flieBenden Welle") kam es mit Ausnahme des in Abschnitt 3.1 erwähnten extremen
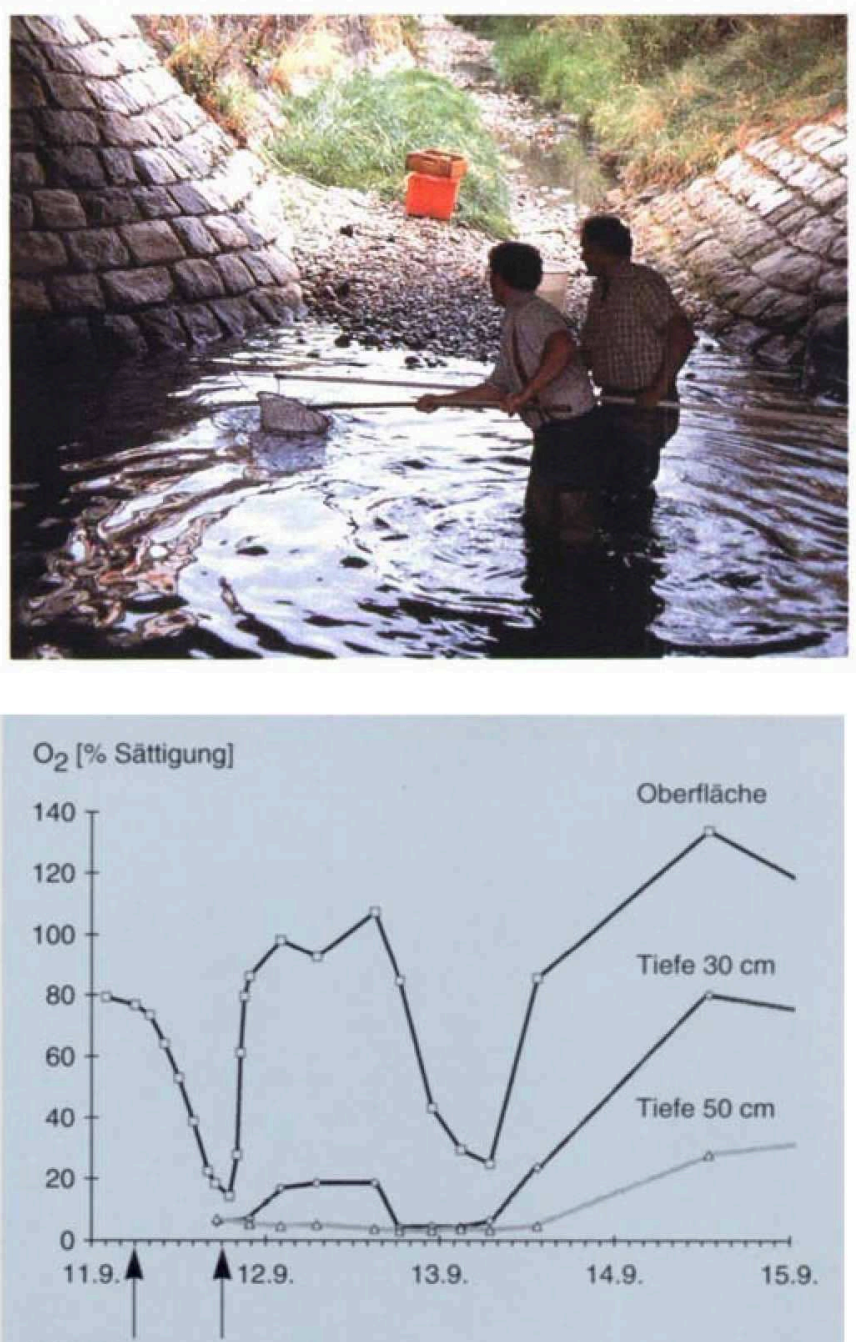

$\mathrm{Q}$ [Ls]

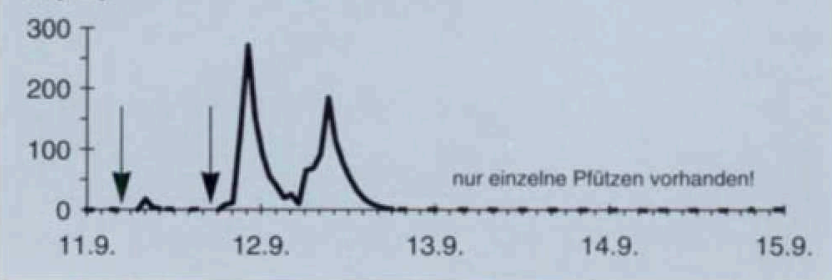

Figur 5. a) Fischereiaufseher beim Abfischen der verendenden Bachforellen nach dem Überlauf-Ereignis vom 11. September 1991 im Hintergrund die ausgetrocknete Luppmen. b) Ganglinien der Sauerstoffsättigung $\left(\mathrm{O}_{2}\right)$ in der Luppmen unterhalb des zweiten Mischwasserüberlaufs (MÜ 2) nach dem Extremereignis vom 11. September 1991. Darunter ist die entsprechende Abflußkurve (Q) eingezeichnet.

Die Überlauf-Ereignisse sind durch Pfeile angegeben: Dem im Text beschriebenen Ereignis folgte am Abend desselben Tages ein zweites, das durch einen stärkeren Regen verursacht wurde. (Die dritte Abflußspitze am Morgen des 12. September rührt von einem Gewitterregen her, der sich nur über das obere Einzugsgebiet der Luppmen ergoß.) Nach dem ersten ÜberlaufEreignis setzte in den Mischwasserpfützen und im darunterliegenden Interstitial eine starke Sauerstoffzehrung ein, durch Bakterien, die organische Bestandteile des Abwassers abbauten. Die nachfolgenden Abflußspitzen bewirkten keine Spülung des Interstitials, sondern nur einen kurzfristigen Anstieg der Sauerstoffsättigung im Oberflächenwasser. Erst drei Tage nach dem ersten Ereignis näherte sich der $\mathrm{O}_{2}$-Gehalt wieder den "normalen" Werten wegen fehlender Strömung erfolgte der $\mathrm{O}_{2}$-Eintrag nur sehr langsam (durch Diffusion aus der Atmosphäre). 
Ereignisses nie zu einem signifikanten Rückgang der Sauerstoffsättigung. Ein akuter Sauerstoffmangel infolge eines Überlauf-Ereignisses als Ursache für eine Schädigung der Biozönose auf der Bachsohle kann daher ausgeschlossen werden. Dagegen scheint die Interstitialfauna stärker gefährdet: Die $\mathrm{O}_{2}$-Messungen an Interstitialwasser während und nach dem erwähnten Extremereignis deuten darauf hin, daß sich Überlauf-Ereignisse bis in eine Tiefe von 50 (!) Zentimeter oder mehr auswirken können, wenn die Umstände ein Absetzen oder Einschwemmen des feinteiligen organischen Materials ermöglichen. Ein uiber mehrere Tage andauernder Sauerstoffschwund, wie er in der Figur 5b dargestellt ist, wäre für die Interstitialfauna des betroffenen Bachabschnitts katastrophal: Die Tiere würden sich entweder von der Strömung abtreiben lassen oder würden ersticken. Im betrachteten Fall zeigten sich aber kaum biologische Wirkungen, weil die Strecke, wie schon erwähnt, wegen der vorangegangenen, langanhaltenden Trockenheit sowieso nicht mehr von Fließwasserorganismen besiedelt gewesen war.

Es ist zu vermuten, daß auch bei weniger extremen Überlauf-Ereignissen eine Sauerstoffzehrung im Interstitial stattfindet. Dies muß aber noch durch entsprechende Messungen bestätigt werden.

\subsection{Temperatur und $\mathrm{pH}$}

Ein Überlauf-Ereignis kann zu einer sprunghaften Veränderung der Temperatur und des $\mathrm{pH}$-Werts im Vorfluter führen. In der Luppmen, deren Wasser an dieser Stelle einen großen Anteil Grundwasser enthält (kühl, $\mathrm{CO}_{2}$-reich, daher niedriger pH-Wert), erhöhten sich im Sommer 1991 beide Parameter nach einem derartigen Ereignis drastisch (Figur 6). Ob und wie die Benthosorganismen auf solche Veränderungen reagieren, wurde bisher nicht untersucht. Erhebliche Reaktionen können aber nicht ausgeschlossen werden. So ist zum Beispiel bekannt, daß der Strudelwurm Crenobia alpina doppelt soviel Sauerstoff verbraucht, wenn im Bereich von 10 bis $15^{\circ} \mathrm{C}$ die Temperatur nur um $2{ }^{\circ} \mathrm{C}$ erhöht wird ${ }^{[13]}$.

\section{Biologische Befunde}

\subsection{Drei Stellen im Vergleich}

Um es gleich vorwegzunehmen: Während der fast zwei Jahre dauernden, intensiven biologischen Untersuchung

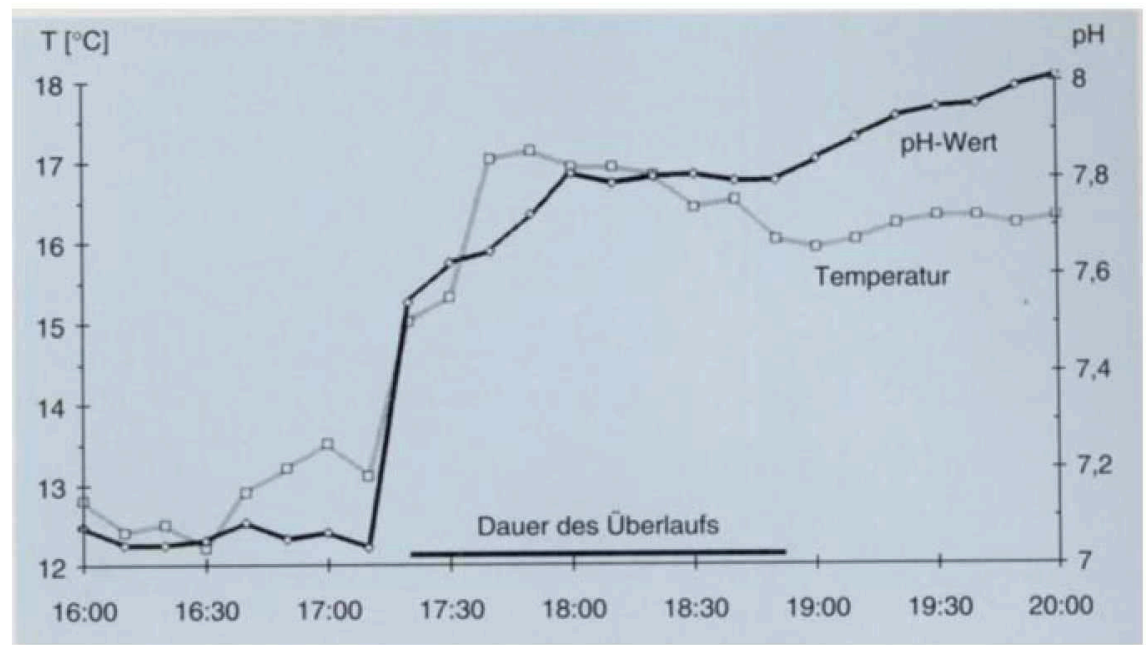

Figur 6. Temperatur- und pH-Werte unterhalb des zweiten Mischwasserüberlaufs (MÜ 2) am 24. Juli 1991. Da die Luppmen an dieser Stelle von kühlem, $\mathrm{CO}_{2}$-reichem Grundwasser (Temperatur $12^{\circ} \mathrm{C}, \mathrm{pH} 7.0$ ) beeinflußt wird, sind die Veränderungen nach Anspringen des Überlaufs besonders drastisch. konnten keine wesentlichen Unterschiede zwischen den Stellen oberhalb ("Stelle 2") und unterhalb des Mischwasserüberlaufs ("Stelle 3") entdeckt werden. Auffallende Differenzen bestehen jedoch zwischen den beiden Fehraltorfer Stellen (im Abschnitt III) und derjenigen der Referenzstrecke ("Stelle 1" im Abschnitt 1; siehe Figur 7).

Obwohl sich die Stellen 2 und 3 infolge der Verbauung der Luppmen in Strömungsgeschwindigkeit und Wassertiefe merklich unterscheiden (die Strömung ist an Stelle 2 durch den Einbau von Querschwellen viel geringer), weisen beide eine sehr ähnliche Biozönose auf. Alle in mehr als 6 Prozent der Proben (und damit nicht rein "zufällig") vorkom- menden Taxa werden an beiden Stellen gefunden, wenn auch zum Teil in unterschiedlicher Dichte. Dasselbe gilt für die zeitliche Dynamik der Besiedlungsdichte, wie das Beispiel der Eintagsfliege Habroleptoides modesta zeigt (vergleiche Figur 9 auf Seite 223). Langzeitschäden als Folge der Einleitung von Mischwasser scheinen also keine zu bestehen.

Gewisse Unterschiede beobachtet man man lediglich in der Häufigkeit der verschiedenen Taxa: Asellus aquaticus (Wasserassel), die Steinfliegen Nemoura sp. und Chloroperla tripunctata sowie die Eintagsfliege Caenis sp. sind an der Stelle 2 (oberhalb MÜ) häufiger, während an der Stelle 3 (unterhalb MÜ) die Flohkrebse Gammarus fossarum und Gam-

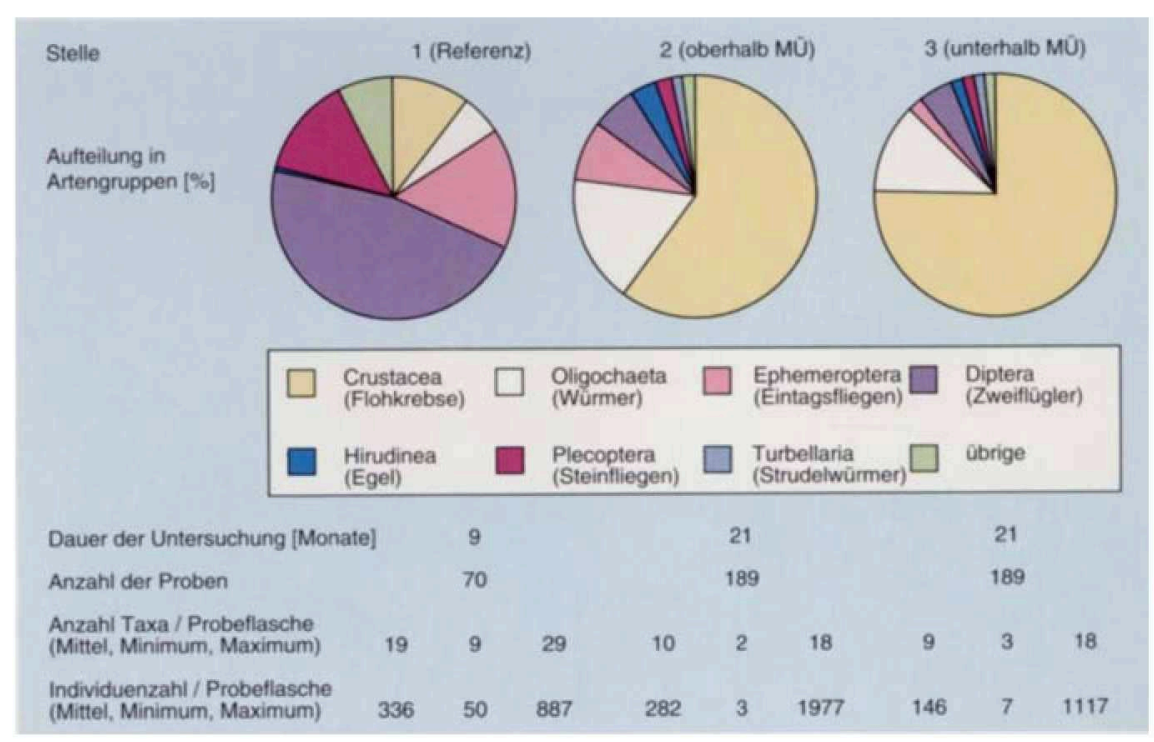

Figur 7. Zusammenfassung der Ergebnisse der biologischen Untersuchungen an den drei Probestellen (vergleiche Figur 4). 
marus pulex, der Wurm Eiseniella tetraedra, die Strudelwürmer Dugesia lugubris und Dugesia gonocephala und die Insektenlarven Leuctra fusca (eine Steinfliege), Tabanus sp. (Bremsenlarve), Vertreter der Familie Ceratopogonidae (Gnitzen), Limnius sp. (ein Käfer) und Odontocerum albicorne (eine Köcherfliege) häufiger sind.

\section{2. "Nutznießer" der Überlauf-Ereignisse}

Die Frage, ob diese Unterschiede auf den Einfluß des Mischwassers zurückzuführen sind, oder ob andere Faktoren, wie die ungleiche Strömungsgeschwindigkeit oder Wassertiefe, dafür ausschlaggebend sind, läßt sich nicht pauschal für alle Taxa beantworten. Diejenigen Arten, welche unterhalb eines Mischwasserüberlaufs deutlich häufiger sind als oberhalb, scheinen in irgendeiner Weise von den Überlauf-Ereignissen profitieren zu können. Für drei Tiere (Eiseniella tetraedra, Gammarus sp. und Dugesia sp.) kann dies aufgrund der autökologischen Kenntnisse bestätigt werden. Alle übrigen in unterschiedlicher Häufigkeit auftretenden Taxa waren dermaßen selten (immer weniger als 10 Individuen pro Probe), daß eine Interpretation der Daten schwierig ist, zumal keine kausalen Zusammenhänge zwischen der Veränderung in der Besiedlungsdichte und den Überlauf-Ereignissen gefunden werden konnten.

\section{Nutznießer von fein-partikularem, organischem Material (FPOM): \\ Eiseniella tetraedra}

Die größere Häufigkeit von Eiseniella tetraedra unterhalb des Mischwasserüberlaufs rührt vermutlich daher, daß dort viel mehr FPOM abgelagert wird, welches die hauptsächliche Nahrungsquelle dieses Wurms ist. Allerdings sei angemerkt, daß die Gesamtzahl aller Würmer (Oligochaeta) an beiden Beobachtungsstellen etwa gleich ist, obwohl sich auch andere Oligochaeta-Arten von FPOM ernähren.

\section{Nutznießer von grob-partikularem Material: Gammanus sp.}

Im Sommer 1990 konnten kurzfristige Veränderungen der Gammarus-Besiedlung eindeutig mit dem Überlaufen von Mischwasser in Zusammenhang gebracht werden: Drei Überlauf-Ereignisse ohne nachfolgende Abflußspitze führten in der Luppmen unterhalb MÜ 1 jeweils zu gut sichtbaren Ablagerungen auf der Bachsohle, insbesondere von WC-Papier. Dort (nicht aber oberhalb MÜ 1) nahm in der Folge auch der Bestand an Gammarus markant zu (Figur 8). Da die Bachflohkrebse als effiziente Zerkleinerer von Detritus aller Art (warum nicht auch WCPapier?) bekannt sind, läßt sich dieses Phänomen wie folgt erklären: Normalerweise ernährt sich Gammarus von Laub, abgestorbenen Algen und Wasserpflanzen, auch von toten oder verletzten Tieren. Bei Nahrungsmangel machen sie aber auch vor dem eigenen Nachwuchs nicht halt. Die Fortpflanzungssaison dauert in der Regel von April bis Oktober und erreicht ihren Höhepunkt in den wärmsten Sommermonaten, wobei ein Weibchen hintereinander mehrere Bruten austragen kann Bis zum Zeitpunkt des ersten ÜberlaufEreignisses am 30. Juli nahm die Zahl der adulten Gammariden nur geringfügig zu, obwohl dauernd Junge produziert wurden. Da keinerlei Detritus im Bachbett vorhanden war - ein langdauerndes Hochwasser im Juni hatte den Algenbewuchs abrasiert und den Bach vollständig

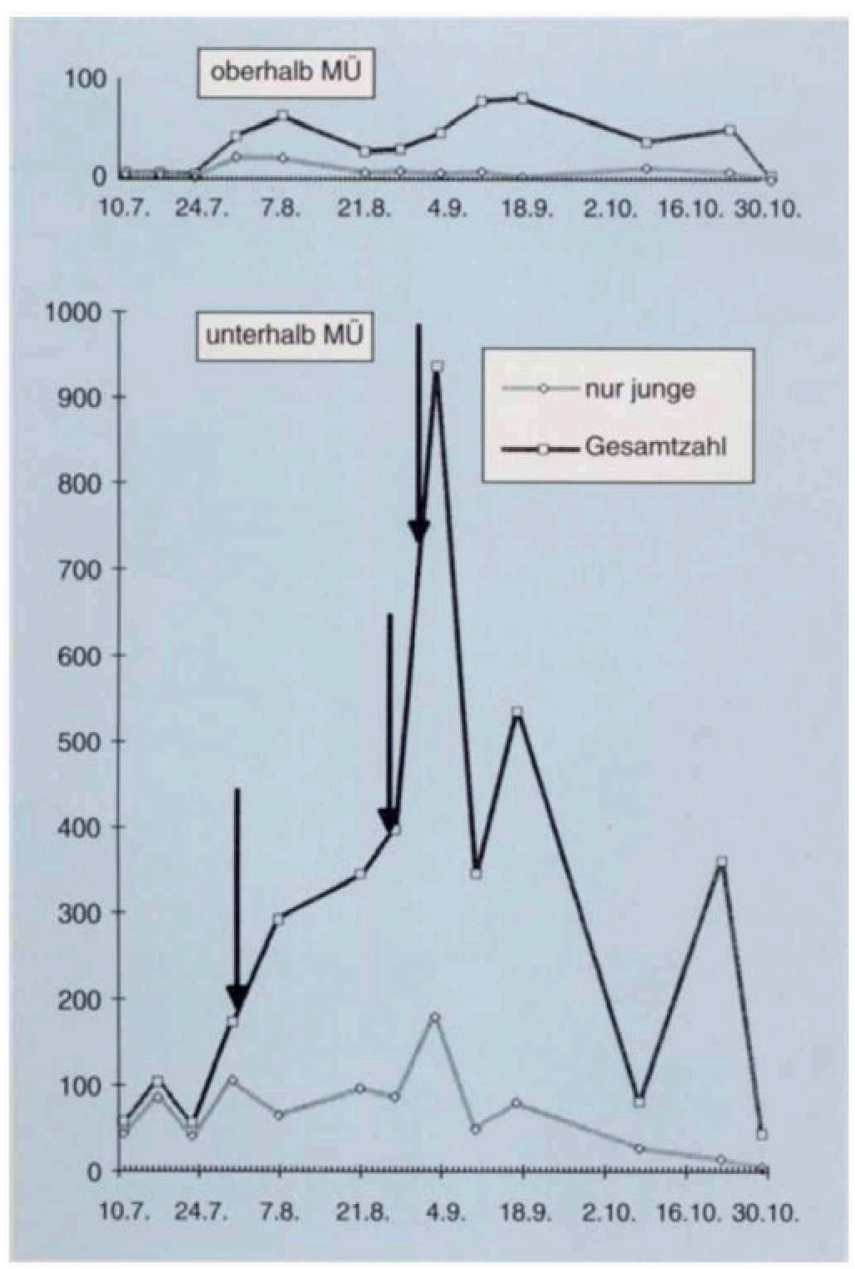

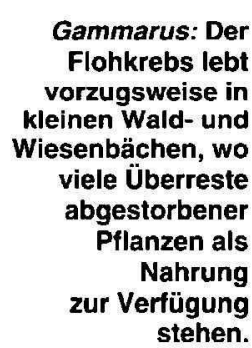

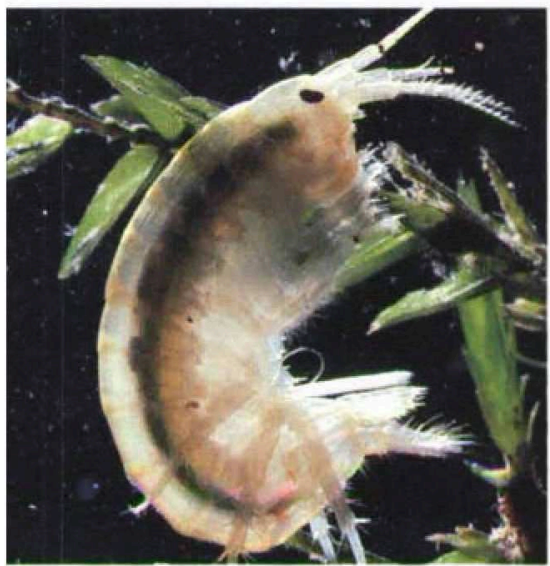

Figur 8.

Entwicklung der Bachflohkrebse

Gammarus fossarum und Gammarus pulex: Besiedlungsdichte

(Anzahl Tiere pro

Probeflasche)

oberhalb und

unterhalb

des ersten

Mischwasser-

überlaufs (MÜ 1;

Juli bis Oktober 1990

Mittelwerte aus drei

Proben).

Die Zeitpunkte der

Überlauf-Ereignisse

sind durch Pfeile

markiert. "ausgeräumt" - ernährten sich die ausgehungerten Alten vermutlich vorwiegend von ihrem eigenen Nachwuchs. Nachdem durch die Überlauf-Ereignisse reichlich "Detritus" in den Bach eingetragen worden war, wechselten die Tiere auf diese Nahrungsquelle über, und die Brut konnte nun ungestört aufwachsen. Dies äußerte sich in einer markanten Zunahme der Gammarus-Abundanz. Dasselbe Phänomen zeigte sich knapp einen Monat später noch einmal nach zwei weiteren Überlauf-Ereignissen. Dann gingen die Zahlen wieder stark zurück; vermutlich verteilten sich die Tiere im Laufe der Zeit auf größere Bachabschnitte. 
Nutznießer von Gammarus: Dugesia sp.

Von Dugesia lugubris und Dugesia gonocephala, zwei räuberisch lebenden Strudelwürmern, ist bekannt ${ }^{[14]}, \mathrm{da} ß$ sie unter anderem auch verletzte Gammariden (Bachflohkrebse) angreifen und aussaugen. Da die dichtesten Dugesia-Besiedlungen jeweils kurz nach den "GammarusMaxima" auftraten, welche ihrerseits mit den Überlauf-Ereignissen erklärt werden können (siehe oben), scheint die Besiedlungsdichte von Dugesia primär von einem synökologischen Mechanismus (Räuber-Beute-Beziehung) abzuhängen.

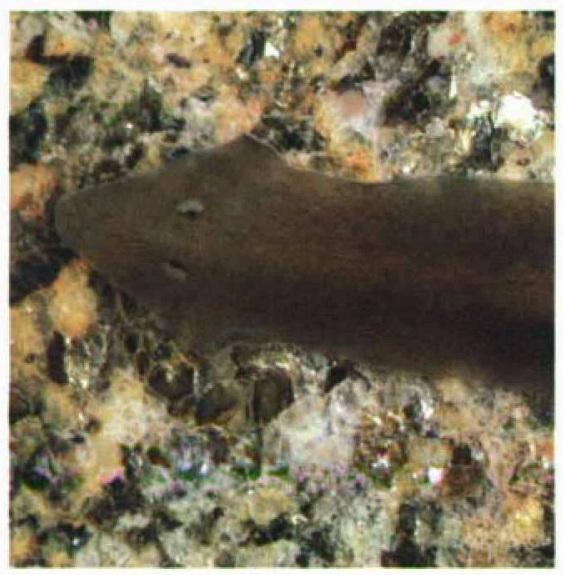

Dugesia: Die Strudelwürmer ernähren sich räuberisch von anderen Kleinlebewesen. Die verschiedenen Arten stellen sehr unterschiedliche Ansprüche an ihre Umgebung: während manche in warmen und mäßig verschmutzten Bächen vorkommen, findet man andere nur in kalten, sauberen Quellen und Gebirgsbächen.

\subsection{Biologische Gesamtbeurteilung der Luppmen: Wo sind die Schäden?}

Bisher wurde nur von Organismen berichtet, welche von den MischwasserEinflüssen profitieren. Ein Verschwinden empfindlicher Organismen als Folge eines Überlauf-Ereignisses konnte nie beobachtet werden. Ein wichtiger Grund hierfür dürfte sein, daß einige typische Fließwasserorganismen im Abschnitt III schon an der Stelle 2 (oberhalb MÜ) gar nicht mehr vorkommen. So fehlen zum Beispiel die netzbauenden Köcherfliegenlarven der Gattung Hydropsyche, die Eintagsfliegenlarven Ephemera danica, Paraleptophlebia submarginata und Ecdyonurus venosus, der Strudelwurm Polycelis nigra sowie etliche andere Arten, welche einige Kilometer weiter oben, im weitgehend natürlich gebliebenen Teil der Luppmen (Stelle 1), recht häufig sind. Alle diese Organismen sind in irgendeiner Weise hochspezialisiert, sei es auf eine bestimmte Nahrungsquelle (Hydropsyche sp.), eine bestimmte Korngröße des . Substrats (Ephemera danica) oder eine bestimmte Strömungsgeschwindigkeit (Ecdyonurus venosus, Paraleptophlebia submargina$t^{\left[{ }^{[15}\right)}$. Die Verbauung der Luppmen in den Abschnitten $I I$ und $I I I$ hat jedoch zur Folge, daß die ökologischen Nischen dieser Tiere eingeengt werden oder ganz verschwinden: Das begradigte, von jeglichen Hindernissen befreite, eingeebnete Bachbett läßt keine vielfältigen Strömungsmuster entstehen. Es bietet weder strömungsgeschützte Rückzugsräume bei Hochwasser noch garantiert es bei niedriger Wasserführung an gewissen Stellen eine ausreichende Fließgeschwindigkeit. Die Sohlenstabilisierung bewirkt, daß bei Hochwasser trotz sehr hoher Strömungsgeschwindigkeiten $(>1 \mathrm{~m} / \mathrm{s} 5 \mathrm{~cm}$ über der Sohle!) kein Aufbrechen der Deckschicht und damit praktisch keine Umlagerung des Sohlenmaterials mehr stattfindet. So wird die Sohle allmählich immer fester, und die Porenräume des Interstitials verstopfen (Kolmation). Da die teils mit Steinplatten befestigten, im Aufriß trapezförmigen Ufer kaum mit Gehölzen bewachsen sind, ist der Bach noch dazu der vollen Sonneneinstrahlung ausgesetzt. Dadurch werden im Sommer oft sehr hohe Temperaturen (Maxima über $25^{\circ} \mathrm{C}$ ) erreicht, die für viele Fließwassertiere bereits tödlich sind.

Eine Gegenüberstellung des biologischen Aspekts aller drei Untersuchungsstellen ergibt somit, daß die Biozönose der Luppmen bereits oberhalb des ersten Mischwasserüberlaufs durch die morphologischen Eingriffe und die Effekte der Wasserentnahme stark geschädigt ist. Die wenigen Arten, welche sich trotzdem im Gewässer halten können ("Rumpfbiozönose"), sind derart unempfindliche "Generalisten", daß ihnen auch die Zuflüsse von Mischwasser kaum etwas anhaben können. Einige Befunde sollen dies verdeutlichen (vergleiche Figur 7):

- Im naturnahen Teil der Luppmen (Stelle 1) wurden bisher schon 67 Taxa gefunden, obwohl diese Strecke erst seit 9 Monaten untersucht wird. Hingegen ergab die seit 21 Monaten andauernde Beobachtung der Stelle 2 (oberhalb MÜ 1) nur 51 Taxa, diejenige der Stelle 3 (unterhalb MÜ 1) 48 Taxa.

- An der Referenzstelle werden in einer einzelnen Probe im Durchschnitt rund doppelt so viele Taxa gefunden wie an den Stellen 2 und 3.

- Die Individuendichte ist an der Stelle 1 im Mittel höher und schwankt um einen wesentlich geringeren Betrag als an den beiden anderen Stellen.

Ein wesentlicher Grund für diese Situation dürfte sein, daß sich an der Stelle 1 harsche Umweltbedingungen wie extrem hohe oder niedrige Abflüsse viel weniger stark auf die Besiedlung auswirken als an den anderen Stellen, da für die Fauna genügend Rückzugsräume offenstehen. Ins Wasser ragende Baumwurzeln, Felsblöcke und Kiesbänke, Kolke und vor allem ein lockeres Sediment puffern die äußeren Einflüsse ab. Bei Hochwasser kann der Bach einen wesentlich breiteren Sohlenbereich überfluten, so daß hohe Strömungsgeschwindigkeiten nur punktuell auftreten; an andern Stellen wird das Wasser durch verschiedenste Hindernisse abgebremst. Bei extremem Niedrigwasser zieht sich der Bach in eine schmale, etwas eingetiefte Rinne zurück. Im Abschnitt III dagegen variiert bei unterschiedlicher Wasserführung nur die Wassertiefe, da die Sohle eingeebnet und die Bettbreite praktisch konstant ist.

Für das Verschwinden der einzelnen Tiergruppen oder Tierarten gibt es wiederum keine pauschale Erklärung, sondern sie ist jeweils in den spezifischen Bedürfnissen der Art oder der Tiergruppe zu suchen.

\section{Hochwasserscheu: Die Eintagsfliege Habroleptoides}

Wie katastrophal sich die Mängel an Strukturelementen im Einzelfall auswirken können, zeigt das Beispiel der gegen Mischwasser anscheinend weitgehend unempfindlichen Eintagsfliege Habroleptoides modesta (Figur 9 und 10). Während diese Tiere im Sommer 1990 in großer Zahl als kleine Larven erschienen und im Laufe der folgenden Monate heranwuchsen, blieben die Junglarven im Sommer 1991 an den Stellen 2 und 3 vollkommen aus, während die Entwicklung

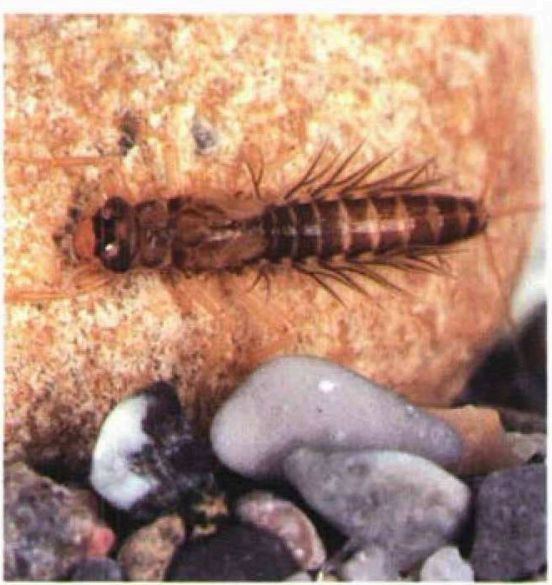

Habroleptoides: Die Larve der Eintagsfliege Habroleptoides kommt in kleinen und mittelgroßen Fließgewässern vor. Sie ernährt sich von feinem Detritus (Schwebe- und Sinkstoffe aus abgestorbenem organischem Material). 


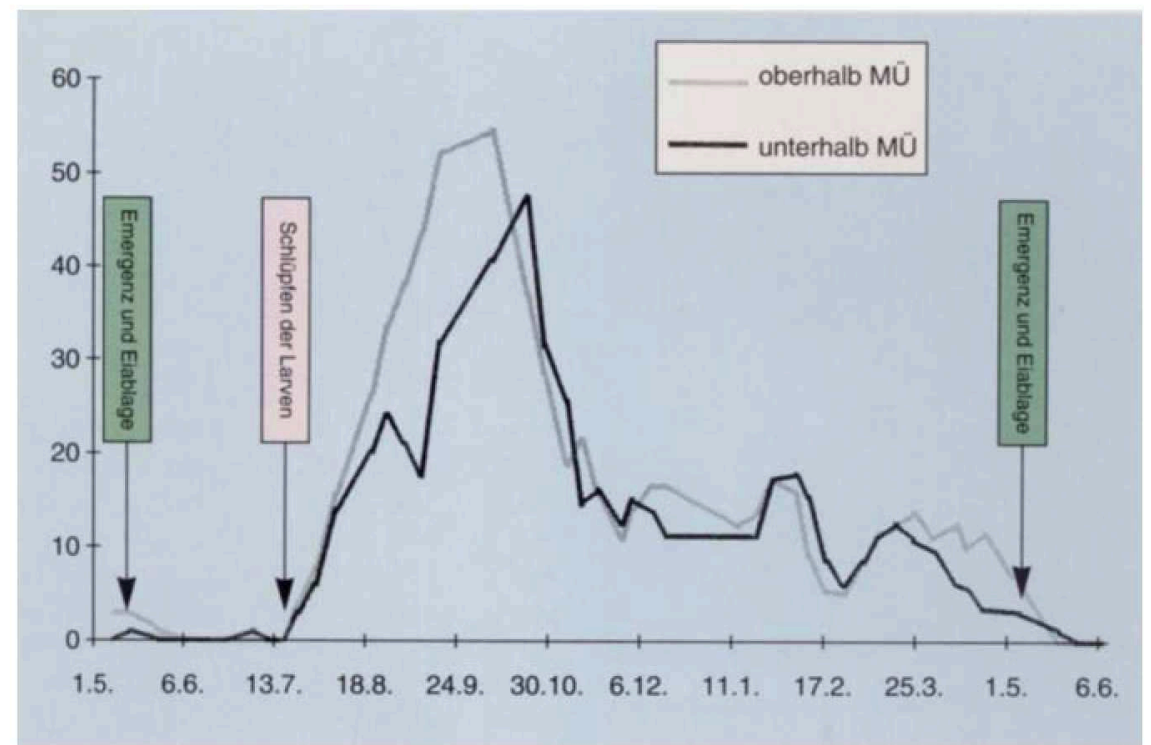

Figur 9. Entwicklung der Eintagsfliegenlarve Habroleptoides modesta: Besiedlungsdichte (Anzahl Tiere pro Probeflasche) oberhalb und unterhalb des ersten Mischwasserüberlaufs (MÜ 1; Mai 1990 bis Juni 1991, Mittelwerte aus drei Proben).

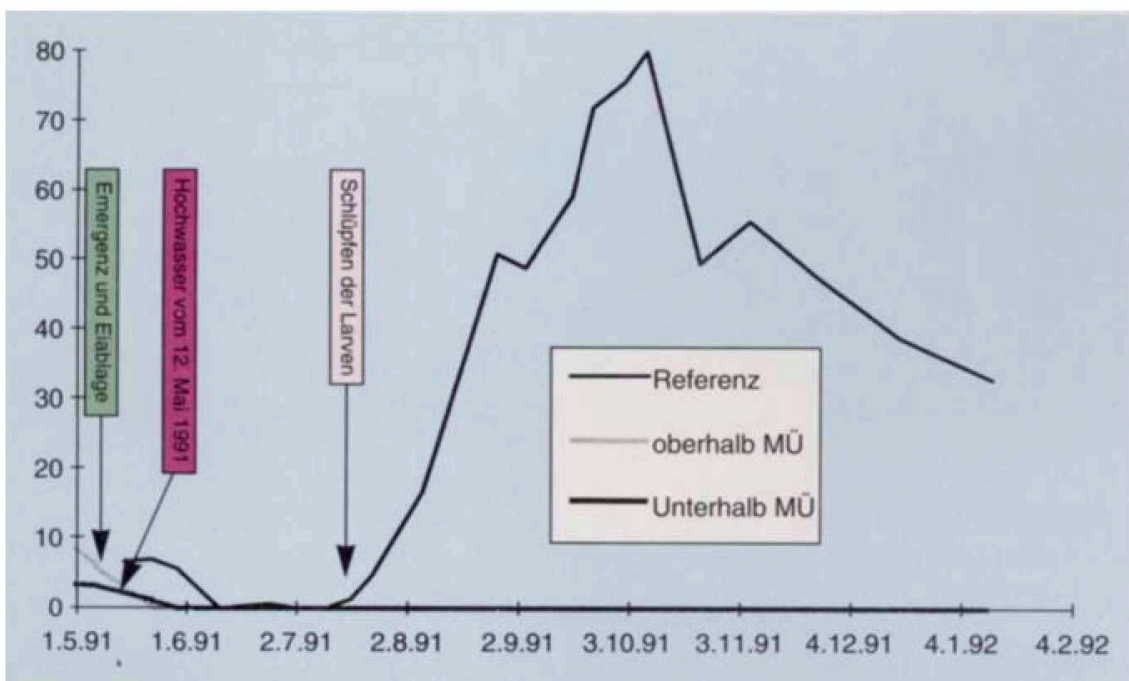

Figur 10. Entwicklung von Habroleptoides modesta im Vergleich mit der Referenzstelle (Anzahl Tiere pro Probeflasche; Mai 1991 bis Januar 1992, Mittelwerte aus drei Proben).

an der Referenzstelle ganz normal verlief. Ein größeres Hochwasser während der Emergenz- und Eiablagephase (April/Mai) hatte sowohl die schlüpreifen Larven als auch die bereits abgelegten Eier die beiden empfindlichsten Stadien im Lebenszyklus dieser Tiere ${ }^{[15]}$ - praktisch vollständig vernichtet. Bezeichnenderweise geschah dies jedoch nur an den verbauten Stellen, wo die Tiere keine Möglichkeit hatten, sich in die tieferen Sedimentschichten oder an andere, strömungsgeschützte Orte zurückzuziehen. An der Referenzstelle zeigte dasselbe Hochwasser überhaupt keine Wirkung. Auch andere Populationen wie zum Beispiel diejenige der nahe verwandten hochspezialisierte Algenabweider auf und unter den Steinen der obersten Sohlenschicht. Diese Anpassung geht so weit, daß sie auf eine ständige Zufuhr von frischem Wasser angewiesen sind, um ihren vergleichsweise hohen Sauerstoffbedarf zu stillen, und ohne Strömung nicht mehr leben können. Bei ungenügender Strömung ersticken sie innerhalb kurzer Zeit (Minuten). Da das Wasser mit zunehmender Temperatur immer weniger Sauerstoff enthält, die Tiere jedoch für ihren Stoffwechsel immer mehr Sauerstoff verbrauchen, kann die Situation bei niedriger Wasserführung im Sommer für Ecdyonurus und andere, ähnlich adaptierte Tiere letal werden. Sie verschwinden deshalb aus zu warmen und vor allem zu

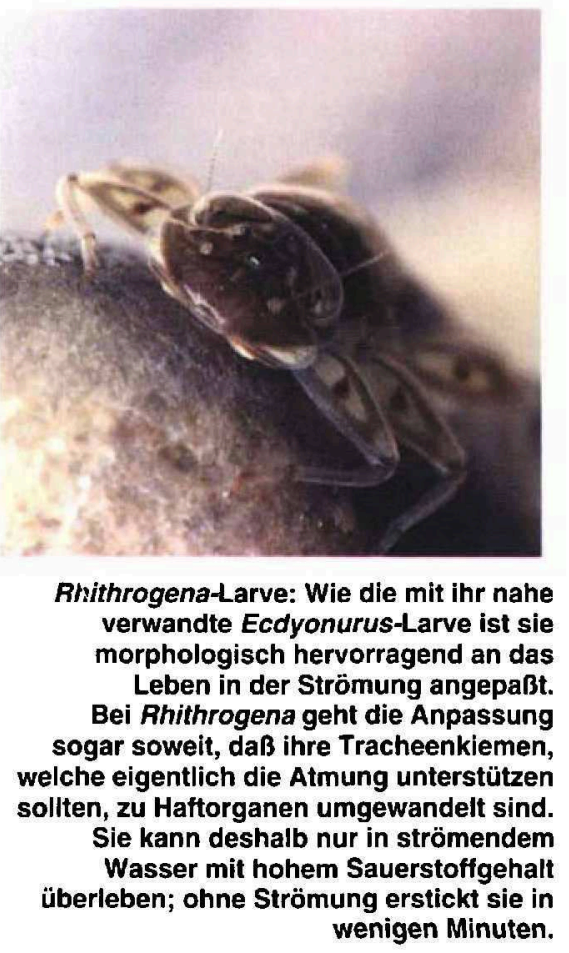

langsam fließenden Gewässerabschnitten. So findet man die Ecdyonurus-Larven im naturnahen Abschnitt der Luppmen stets in großer Zahl. Im Fehraltorfer Abschnitt hingegen kommen sie nur noch ganz lokal unterhalb von (kühlen!) Drainageund Grundwassereinleitungen vor. An den Stellen 2 und 3 wurden nur im Winter einzelne Exemplare gefunden.

\section{Benötigt viel Sauerstoff und starke Strömung: Ecdyonumus}

Andere Probleme als zu hohe Strömungsgeschwindigkeiten hat die Eintagsfliegenlarve Ecdyonurus venosus. Von diesen Tieren halten sich nur die kleinsten Larvenstadien im Interstitial auf; die größeren Individuen sind hervorragend an starke Strömungen angepaßt und leben als

\section{Braucht feinen Sand: Ephemera}

Überall da, wo sich an strömungsgeschützten Stellen feiner Sand ablagern kann, zum Beispiel hinter großen Steinen und zwischen Baumwurzeln nahe der Uferböschung, leben die Larven der Eintagsfliege Ephemera danica. Mit ihren zu 


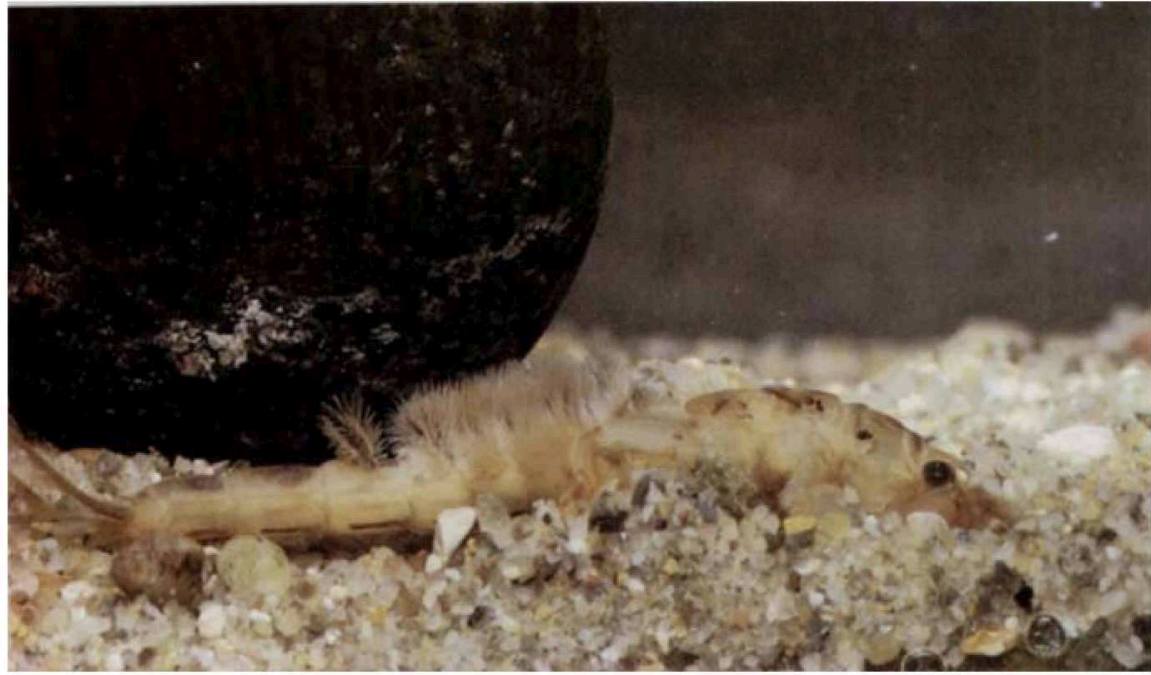

Ephemera: Die Eintagsfliege Ephemera danica lebt als Larve nur dort in Fließgewässern, wo sich feiner Sand abgelagert hat (zum Beispiel im Uferbereich). Ihre Kiefer und Vorderbeine sind, ähnlich wie bei der Maulwurfsgrille (Gryllotalpa gryllotalpa), zu Grabewerkzeugen umgewandelt, mit denen sie sich im weichen Substrat eingräbt. Damit sie genügend Sauerstoff zum Atmen erhält, sind ihre Tracheenkiemen auf der Oberseite des Hinterleibes angeordnet. Ephemera danica, deren Entwicklungszyklus drei Jahre dauert, ist die größte bei uns vorkommende Eintagsfliegen-Art. Ausgewachsene Larven sind etwa $30 \mathrm{~mm}$ lang (ohne Antennen und Schwanzanhänge).

Grabewerkzeugen umgewandelten Beinen und Mundwerkzeugen wühlen sie sich durch den lockeren Sand, auf der Suche nach verwertbarem organischem Material. In der begradigten Luppmen befinden sich, bedingt durch die Gleichförmigkeit der Sohle, keine solchen Sandablagerungen. Deswegen kann auch Ephemera dort nicht mehr existieren.

\section{Auf filtrierbare Partikein angewiesen: Hydropsyche}

Die Köcherfliegen der Gattung Hydropsyche kommen praktisch in allen Fließgewässern des Schweizer Mittellandes vor. Ihre Spezialisierung besteht vor allem in einer raffinierten Art der Nahrungsbeschaffung: Aus einem Speicheldrüsensekret spinnen sie etwa fingernagelgroße Netzchen, die sie zwischen festen Strukturen am Flußgrund aufspannen. Mit diesen Netzchen filtrieren sie Nahrungspartikeln aus dem Wasser. Ihre Häufigkeit ist daher vor allem abhängig vom Nahrungsangebot. Wo stets filtrierbare Partikeln in großen Mengen vorhanden sind, etwa im Ausfluß eines Sees oder Teiches (dort Plankton), aber auch unterhalb von Kläranlagen, beobachtet man stets auch eine dichte Besiedlung mit Hrydropsyche-Larven. Da in der Luppmen der Wasser- und damit auch der Partikeltransport durch das Austrocknen des Abschnitts II oft unterbrochen und das Wasser des Drainagegrabens "Lechbach" weitgehend partikelfrei ist (Bodenfilter!), finden die Hydropsychen im Abschnitt III keine Nahrungsgrundlage; erst unterhalb der Kläranlage Fehraltorf kommen sie wieder in ansehnlicher Zahl vor.

\section{Schlußfolgerungen und Ausblick}

Eines geht aus den bisher vorliegenden Resultaten der Fehraltorf-Untersuchungen ganz klar hervor: Mischwassereinleitung ist nur einer von zahlreichen Faktoren, welche auf die Lebensgemeinschaft eines Fließgewässers einwirken. Weitere wich- tige Faktoren sind unter anderem die Belastungssituation bei Trockenwetter, die Abflußdynamik, die zeitliche und räumliche Heterogenität der Gewässersohle, der Strömungsverteilung und der Wassertiefe, die Morphologie und Linienführung des Gewässers, die Nutzungsart des angrenzenden Landes, der Lichteinfall oder die Beschattung und vieles mehr. Jede einzelne Art der benthischen Lebensgemeinschaft wird durch eine ganz spezifische Kombination dieser Faktoren gefördert oder gehemmt. Alle Einflüsse, welche im Laufe der Entwicklungszeit einer Art auf diese wirken, entscheiden mit, ob sie an einer Stelle überleben kann oder nicht. Nur wenn während der ganzen Entwicklungsdauer alle ihre Umweltbedürfnisse erfüllt werden, kann sie an der Stelle überleben.

Von den meisten Fließwasserorganismen sind die artspezifischen Ansprüche an die natürlichen Parameter (Strömung, Temperatur, Substrattypus, Ufervegetation, Nahrung und so weiter) nicht oder nur sehr ungenau bekannt. Thre Kenntnis ist aber eine Voraussetzung für Aussagen über die Wirkung zusätzlicher, anthropogener Einflußgrößen - wie etwa Mischwassereinleitungen.

Die Untersuchungen im Rahmen des Projekts. "Fehraltorf" ergaben bisher, $\mathrm{da} 3$ meßbare und für die benthische Biozönose relevante Effekte von Mischwassereinleitungen praktisch nur in Ausnahmesituationen auftreten, vor allem dann, wenn die Luppmen sehr wenig Abfluß hat. Unter diesen Umständen kann es $\mathrm{zu}$ Ablagerungen von Grobstoffen auf der Gewässersohle und, als Folge davon, eventuell zu Sauerstoffzehrung im hypo-

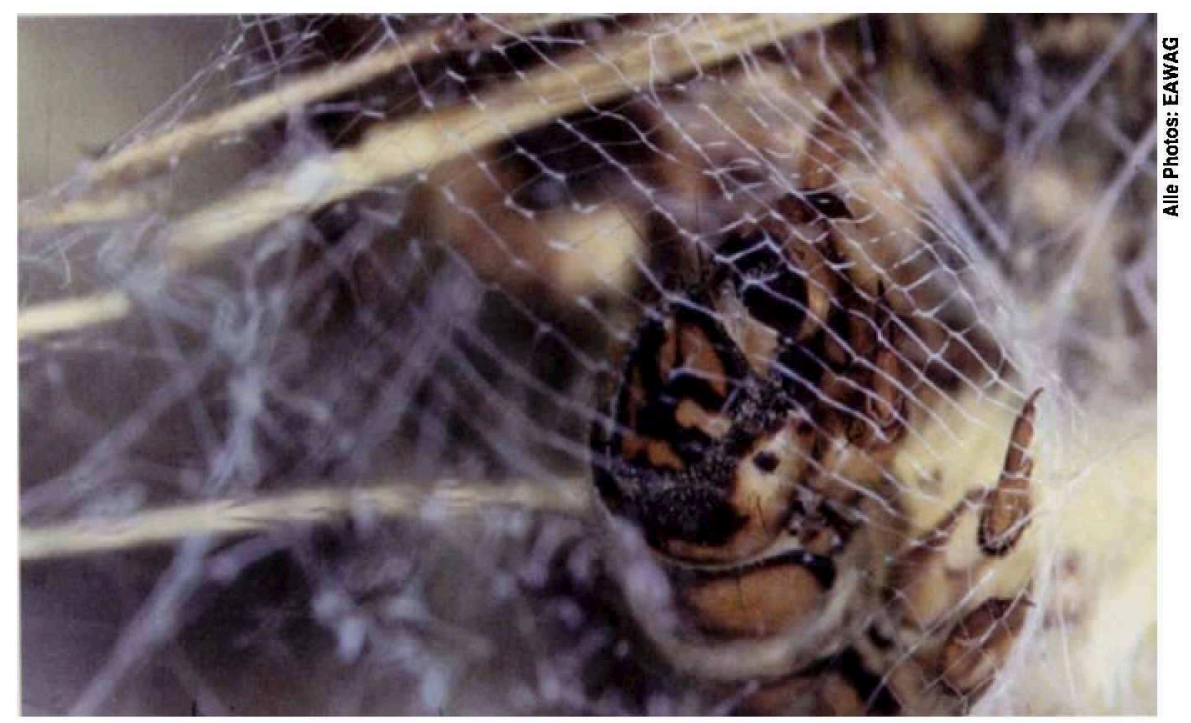

Hydropsyche: Die Larve dieser Köcherfliege spannt zwischen festen Strukturen am Flußgrund ein etwa fingernagelgroßes Netzchen auf, mit dem sie Nahrungspartikeln aus der Strömung filtriert. Wegen dieser Ernährungsweise findet man sie besonders oft dort in den Bächen, wo eine hohe Partikelkonzentration vorkommt, zum Beispiel unterhalb von Kläranlagen (Bakterienflocken) und bei Seeausflüssen (Plankton). 


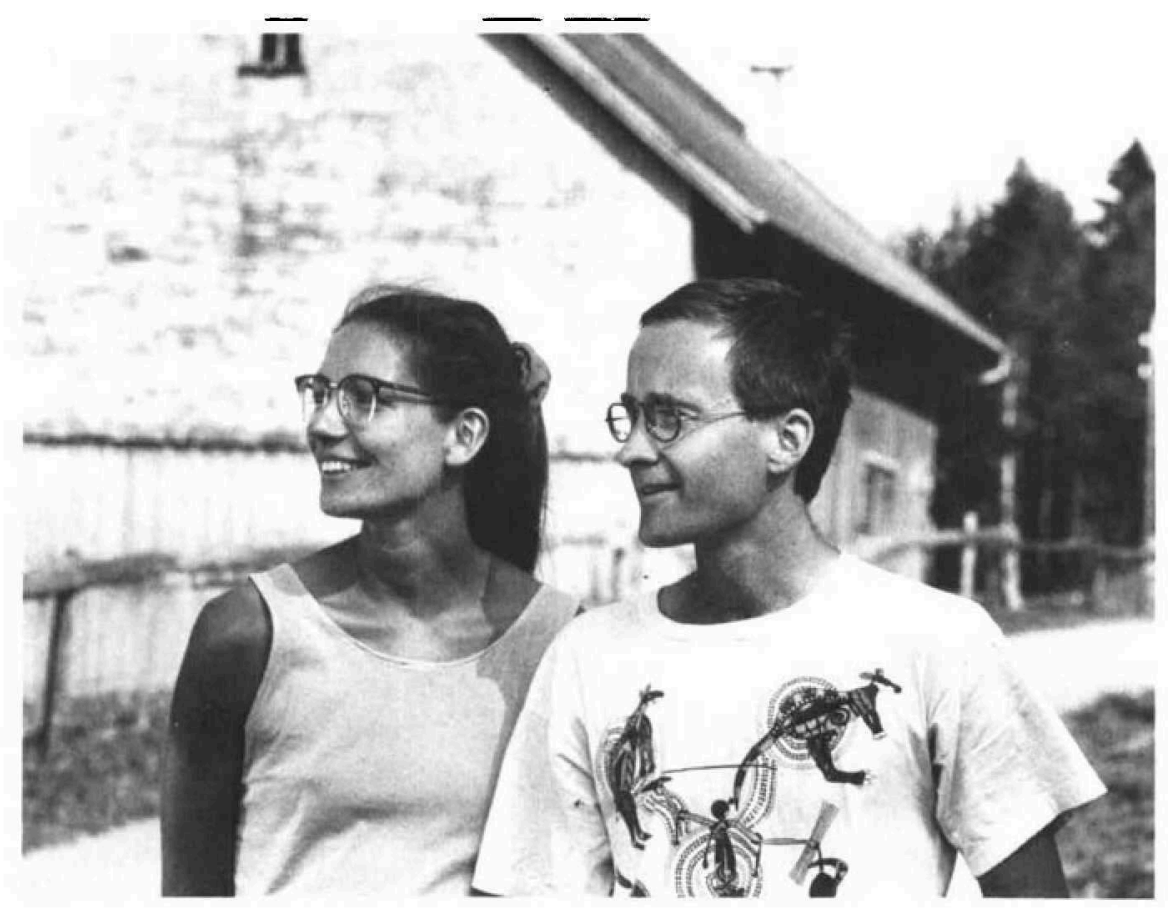

rheischen Interstitial kommen. Allerdings ist hierbei zu berücksichtigen, daß sich unter solchen Bedingungen (sehr wenig Abfluß) auch andere Parameter (zum Beispiel Temperatur, Strömung) verändern und dadurch für die Organismen bedrohliche oder sogar letale Situationen entstehen können, weshalb es wiederum schwierig ist, einen beobachteten Effekt kausal auf eine Mischwassereinleitung zurückzuführen.

Es erscheint aber plausibel, daß in Fließgewässern, welche im Hinblick auf Verbauungsgrad und Organismenbestand mit der Luppmen vergleichbar sind (was für viele "Vorfluter" des schweizerischen Mittellandes zutreffen dürfte), kaum noch nennenswerte Schädigungen durch die Mischwasserüberläufe zu erkennen sind, weil die "Biologie" derartiger Gewässer bereits durch andere Eingriffe, vor allem in die Gewässermorphologie, so verarmt ist, daß die darin noch verbleibende "Rumpfbiozönose" auf die Überlauf-Ereignisse fast gar nicht mehr anspricht.

Bevor die Siedlungsentwässerungs- und Abwasserentsorgungssysteme für viel Geld mit Mischwasserspeichern erweitert werden, nur um die Mischwassereinleitungen zu verhindern, sollte - und zwar für jeden Fall einzeln - der tatsächliche Nutzen eines solchen Speichers durch zielgerichtete biologisch-ökologische Analysen des Gewässers abgeschätzt und mit anderen Gewässerschutzmaßnahmen, zum Beispiel einer Revitalisierung, verglichen werden. Wird das Gewässer auf diese Weise in ein gesamtheitliches Entwässerungskonzept integriert, ist sichergestellt, daß die finanziellen Mittel im Sinne eines umfassenden Gewässerschutzes wirkungsvoll investiert werden. Wenn es darüber hinaus im Bemühen um Revitalisierung gelingt, die durch rigorose Verbauung verlorengegangenen Pufferzonen, Retentionsbereiche und Refugien wieder neu zu schaffen, werden die Fließgewässer auch wieder in die Lage versetzt, ihre vielfältigen Funktionen zu erfüllen - zum Beispiel als Lebensräume einer artenreichen Tierund Pflanzenwelt, als Ausbreitungsachsen amphibischer und terrestrischer Tiere, als ökologische Systeme mit einer hohen Regenerations- und Abbauleistung ("Selbstreinigung") und, last but not least, als Naherholungsräume für den modernen, streßgeplagten Menschen.

\section{Literaturverzeichnis}

[1] A. Hörler: Ingenieur-Handbuch, 78. Auflage, Band II: Kanalisation, Schweizer Verlagshaus AG, Zürich (1966).

[2] V. Krejci, J. Lange, W. Schilling: "Gewässerschutz bei Regenwetter", GAIA l (1992) 72-83.

[3] R. Kolkwitz, M. Marsson: "Ökologie der pflanzlichen Saprobien", Berichte der Deutschen Botanischen Gesellschaft 26a (1908) 505-519;

R. Kolkwitz, M. Marsson: "Ökologie der tierischen Saprobien", Internationale Revue der gesamten Hydrobiologie 2 (1909) 126-152. Schweizerischer Bundesrat, Bern (8.12.1975).

[5] EAWAG, noch nicht veröffentlichte Daten.

[6] C. Belfiore; "Efemerotteri (Ephemeroptera)", Collana del progetto finalizzato "Promozione
[4] Verordnung über Abwassereinleitungen
Sonja Gammeter: Geboren 1964 in Bülach, Kanton Zürich.

Studium der Naturwissenschaften an der Eidgenössischen Technischen Hochschule (ETH) in Zürich; Diplom 1988.

Seit 1989 Mitarbeiterin der Abteilung Ingenieurwissenschaften an der Eidgenössischen Anstalt für Wasserversorgung, Abwasserreinigung und Gewässerschutz (EAWAG) in Dübendorf; Betreuung des biologisch-ökologischen Teils des Projekts "Fehraltorf".

Andreas Frutiger: Geboren 1955 in Bern. Studium der Naturwissenschaften an der Eidgenössischen Technischen Hochschule (ETH) in Zürich; Diplom 1979; Promotion 1983 über die Autökologie einer Steinfliege. 1986-1987 Gastwissenschafter an der University of Canterbury, Christchurch, New Zealand.

Seit 1983 wissenschaftlicher Mitarbeiter de Fachabteilung Hydrobiologie/Limnologie an der Eidgenössischen Anstalt für

Wasserversorgung, Abwasserreinigung und Gewässerschutz (EAWAG) in Dübendorf; Grundlagenforschung und Lehraufträge an der ETH Zürich über Biologie und Ökologie der Fließgewässer. della qualità dell'ambiente", $\mathrm{AQ} / 1 / 20 \mathrm{I}$, Consiglio Nazionale delle Ricerche, Roma (1983), p. 63.

[7] J. M. Elliott, U. H. Humpesch: "A Key to the Adults of the British Ephemeroptera", Scientific Publications of the Freshwater Biological Association, No. 47 (1983), p. 16.

[8] J. Illies: "Ephemeroptera (Eintagsfliegen)", Handbuch der Zoologie, Band 4 (2/7). de Gruyter, Berlin (1968), p. 43, 48.

[9] R. Rawlinson: "Studies on the Life-History and Breeding of Ecdyonurus venosus (Ephemeroptera)", Proceedings of the Zoological Society of London, Series $B$ (1939) 427 ;

H. P. N. Hynes: The Ecology of Running Waters, Liverpool University Press, Liverpool (1970), p. 123.

[10] K. Wuhrmann, H. Woker: "Experimentelle Untersuchungen über die Ammoniak- und Blausäurevergiftungen (Beitrag zur Fischtoxikologie)", Schweizerische Zeitschrift für Hydrologie 15 (1949) 236-260.

[11] S. Gammeter, A. Frutiger: "Wirkung kurzer Belastungsspitzen auf die Kleinlebewesen der Flußsohle", Gas, Wasser, Abwasser 69 (1989) 703-713.

[12] D. Borchardt, B. Statzner: "Ecological Impact of Urban Stormwater Runoff Studied in Experimental Flumes: Population Loss by Drift and Availability of Refugial Space", Aquatic Sciences 52 (1990) 299-314.

[13] B. Statzner: "Fließwasserökologische Aspekte bei der naturnahen Umgestaltung heimischer Bäche", Mitteilungen des Instituts für Wasserbau und Kulturtechnik Karlsruhe 174 (1986) $55 \mathrm{ff}$.

[14] H. Tachet, M. Bournand, P. Richoux: "Introduction à l'étude des Macroinvertébrés des eaux douces", Université de Lyon (1984), p. 157

[15] G. Pleskot: "Zur Ökologie der Leptophlebiiden (Insecta, Ephemeroptera)", Österreichische Zoologische Zeitschrift 4 (1954) 45-107.

(Eingegangen am 25. Juni 1992; mitgeteilt von W. Giger) 